\title{
II. SCHLOSS UND SAMMLUNG IM SCHATTEN MAZARINS FOUQUETS KULTURPATRONAGE DER JAHRE 1653-1655
}

Mit der Übernahme der surintendance des Finances hatte sich auch Fouquets gesellschaftlicher Status geändert. Nach dem Premierminister Mazarin und dem Kanzler Séguier gehörte er nun zu den ersten Männern des Königreichs. Die surintendance des Finances war das höchste Amt, das ein Mitglied seiner Familie je bekleidet hatte. Mit der neuen Position wandelten sich auch die Erwartungen, die man auf öffentlich-repräsentativer Ebene an Nicolas Fouquet stellte. Er leitete von nun an die Staatsfinanzen Frankreichs und musste für die Liquidität der Krone sorgen. In seinem Auftreten galt es, sowohl den Financiers als auch dem Hof Sicherheit zu suggerieren, um nicht nur die Finanzierung des Staatshaushalts, sondern auch die eigene Stellung nicht (doppelt) zu gefährden. Die neuen Anforderungen und Ambitionen übten einen großen Einfluss auf das bauherrliche und mäzenatische Verhalten Fouquets aus, der sich kurz nach der Ernennung zum Finanzminister als Zeichen seiner Rangerhöhung seinem ersten Schlossbauprojekt widmete. Neben dem repräsentativen Wohnsitz in Saint-Mandé trug er seiner neuen Stellung nach 1653 durch den Ausbau der von seinem Vater geerbten Bibliothek, den Aufbau einer Kunstund Kuriositätensammlung und die Förderung von Literaten Rechnung. Mit dem Engagement als Mäzen verfolgte der Finanzminister drei Ziele: in erster Linie diente seine Kulturpatronage der Legitimation und Repräsentation seiner neuen gesellschaftlichen Stellung. Um sich unter den anderen Ministern zu etablieren, musste er auch auf kulturpolitischer Ebene ihren Ansprüchen gerecht werden. Darüber hinaus band Fouquet mit seinem Mäzenatentum die künstlerische und geistige Elite des Landes an sich, die ihm in ihren Werken huldigte und so zum Sprachrohr seines Strebens nach gesellschaftlicher Anerkennung wurde. Nicht zuletzt waren ein repräsentativer Schlossbau und das Engagement als Kulturförderer besonders für einen Finanzminister unentbehrlich. Der Kauf und Ausbau eines Schlosses täuschte den Gläubigern der Krone eine heile finanzielle Welt vor, für die der Finanzminister bürgte.

\section{Der erste Schlossbau: Saint-Mandé}

Aufgewachsen im elterlichen Haus in der Pariser Rue de Jouy, lagen Fouquets Domizile bis 1653 allesamt in der Stadt an der Seine, deren Architektur den Finanzminister geprägt hatte ${ }^{1}$. Im Marais waren im 17. Jahrhundert plan-

1 Am 27. Januar 1615 wurde Fouquet in der maison familiale in der Rue de la Verrerie geboren, zog bald darauf in das Haus in der Rue de Jouy, wo er seine Kindheit verbrachte. 
voll angelegte Gärten und eine Architektur entstanden, die sich durch die Vermischung von antiken Elementen und modernen Bedürfnissen auszeichnete. Das von François Mansart errichtete Hôtel d'Antoine d'Aumont, das sich - wie Nicolas Elternhaus - in der Rue de Jouy befand, gab Fouquet einen ersten Eindruck des Könnens seines späteren Dekorateurs Charles Le Brun. Seitdem der Pont Marie und der Pont de la Tournelle die Île Saint-Louis mit den beiden Ufern der Seine verbanden, ließen sich auch hier zwischen 1635 und 1648 Parlamentsräte und Financiers hôtels und Häuser errichten, unter ihnen das von Louis Le Vau entworfene Hôtel Lambert.

Nachdem er am 8. Februar 1653 in das Amt des surintendant des Finances berufen worden war, bezog Nicolas Fouquet sein erstes repräsentatives Anwesen auf dem Lande. Im März 1654 kaufte er eine Residenz vor den Toren von Paris, um sie als maison de plaisance zu nutzen ${ }^{2}$. In Saint-Mandé erwarb er im Tausch gegen zwei Pariser Häuser in der Rue Saint-Antoine und der Rue de Jouy aus dem Besitz seiner Gattin ein Grundstück, das er in den folgenden Monaten durch den Zukauf von Ländereien und Häusern in der Nachbarschaft vergrößerte ${ }^{3}$. Mit Hilfe Mazarins erhielt er ein königliches Brevet, das ihm die Einfriedung des gesamten Besitzes erlaubte ${ }^{4}$. Die Wahl des Ortes für den neuen Landsitz traf Fouquet nicht willkürlich. Sein neues Schloss Saint-Mandé lag in unmittelbarer Nachbarschaft zu einer königlichen Domäne. Vincennes gehörte zwar nicht zu den beliebtesten Schlössern des jungen Königs, mit der Zeit war es aber zu dem bevorzugten Aufenthaltsort Mazarins vor den Toren der Stadt geworden. Dieser hatte nach der Fronde

Nach seinen Schuljahren bei den Jesuiten im Collège Clermont, Rue Saint-Jacques, bewohnte er ein Haus in der Sackgasse Saint-Thomas (bei der Rue Matignon), war im Jahre 1648 wohnhaft in der Rue des Mauvais-Garçons (BN ms. fr. n. a. 6205, fol.157), kaufte 1650 das sogenannte Hôtel d'Estrée in der Rue Barbette, zog nach seiner Eheschließung mit Marie-Madeleine de Castille im Jahre 1651 in das zwischen der heutigen Rue Michelle-Comte und der Rue Vieille-du-Temple gelegene »vieil hostel de Montmorency que lui apportait en dot sa seconde femme «(BHVP ms. 500, fol.291), 1658 in das Hôtel de Narbonne in der Rue Saint-Honoré (welches er durch einen unterirdischen Gang mit dem hôtel auf der anderen Straßenseite verbinden ließ) und kaufte um 1660 auf Drängen Mazarins das Hôtel d'Émery in der Rue Croix-des-Petits-Champs, dessen Garten einen Teil der heutigen Place des Victoires einnahm.

2 Zur Problematisierung des Begriffs maison de plaisance siehe: Krause, Maison de plaisance, S. 8f. Krause bezeichnet die maison de plaisance als ein Phänomen des 17. Jahrhunderts, welches baulich nur schwer zu definieren ist und sich vielmehr durch seine Nutzung bestimmt. Es ist ein Ort der Vergnügungen, die je nach Bewohner und dessen Stand anders ausfallen können.

${ }^{3}$ Der Tauschvertrag trägt das Datum vom 30. März 1654. Tauschpartner waren Catherine Bellier, erste Kammerfrau Anna von Österreichs, und ihr Gatte Pierre de Beauvais, conseiller d'État (AN MC/ET/LI 534, 30 mars 1654). Zu den weiteren Tauschverträgen siehe auch: MicheL, Vatel, S.38.

4 »L'enceinte [n'est] moindre que M. le Cardinal ne l'avoit dit. Ce fut luy-même qui l'ordonna, il vouloit que j'y joignisse encore d'autres terres au delà du Potager, autant qu'il y en avoit déja d'enclos; Ce fut luy qui fit expedier le Brevet du Roy pour faire la clôture, M. le Secrétaire d'Estat en peut rendre témoignage« (Fouquet, Euvres, Bd. V, S. 139). 
den Titel des gouverneur de Vincennes und damit das Wohnrecht für das Schloss erhalten, das er in den folgenden Jahren unter der Leitung Louis Le Vaus um- und ausbauen ließ $\beta^{5}$. Von seinem neuen Anwesen aus hatte Fouquet unmittelbaren Zutritt zu den königlichen Gärten. Der Kauf von Saint-Mandé war also strategisch günstig und - beachtet man den Zeitpunkt des Erwerbs politisch motiviert. Für den jungen und aufstrebenden Finanzminister, der seinen Karrieresprung der Protektion durch Mazarin verdankte, war es von größter Bedeutung, stets in unmittelbarer Nähe des Mannes zu sein, von dem seine weitere Karriere abhing. Für die Wahl des Ortes sprach zudem, dass auch eine Vertraute des Finanzministers, Mme du Plessis-Bellière, in Charenton, unweit von Saint-Mandé wohnte ${ }^{6}$. Saint-Mandé war also der ideale Ort für den aufstrebenden Finanzminister und zugleich Ausdruck seines politischen Erfolges. Er ermöglichte eine rasche Anreise von Paris und befand sich in unmittelbarer Nähe zu einem der Machtzentren des Königreichs.

In den Jahren bis 1661 wurde das Anwesen zum bevorzugten ländlichen Aufenthaltsort des Finanzministers, an dem er Bittsteller aller Art und - seiner Position gemäß - hohen Besuch empfing. Fouquet nannte eben diese illustren Gäste während seines Prozesses als Grund für den herrschaftlichen Ausbau von Saint-Mandé:

J'ay fait de la dépense à Saint Mandé, c'estoit une maison où je demeurois la plus grande partie de l'année, je l'ay voulu rendre logeable, M. le Cardinal est venu la voir, \& m'a donné le dessein du logement de la closture \& des augmentations à y faire, j'y ay travaillé peu à peu, je n'ay fait aucune dépense à la maison de Paris, j'avois de fort grands revenus, lesquels engagent d'ordinaire à la dépense de ceux qui sont d'une humeur un peu liberale, j'estois visité des personnes les plus considérables du Royaume, j'ay voulu accomoder une maison pour les recevoir, j'y ay fait un jardin, \& je l'ay accompagné des agrémens ordinaires qui sont dans les autres maisons ${ }^{7}$.

Das »château de Saint-Mandé«, wie es in einigen Quellen genannt wird ${ }^{8}$, ist längst verschwunden. In dem Pariser Vorort weist nurmehr eine kleine Tafel an der Rue de l'Épinette auf das ehemalige Anwesen des Finanzministers hin ${ }^{9}$. Wenige Quellen erzählen von dem Aussehen und der Ausstattung SaintMandés. Stiche des Anwesens, die Aufschluss geben könnten über seine bauliche Form, sind nicht überliefert. Erstmals bei Brix abgedruckt ${ }^{10}$ findet sich

\section{Ballon, Louis Le Vau, S. 21f.}

${ }^{6}$ Paulet-Renault, Roture, S.162 führt zudem auf, dass auch einige mit Fouquet zusammenarbeitende Financiers in Charenton ihre Landsitze hatten: »les Malon de Bercy, Pajot d'Ons-en-Bray, Chamillart«.

${ }^{7}$ Fouquet, Cuvres, Bd.X, S.123f.

8 BHVP ms. 374 (Papiers Jarry), fol. 89; Chéruel, Mémoires, Bd. II, S. 180.

9 Ville de Saint-Mandé, rue de l'Épinette: »Simple chemin de terre marquant la limite nord de la propriété du Surintendant Foucquet puis du Couvent des Dames Hospitalières, il fut chassé dans la voirie urbaine en 1904«.

${ }^{10}$ BrIx, Garten, S.44. Eine Kopie des Planes befindet sich zudem in Vaux-le-Vicomte (Erste Etage, im Gang zwischen dem cabinet und der chambre des privaten Appartements von Fouquet). Das Original gehört zur Sammlung Cronstett, Planche 429 des Nationalmuseums von Stockholm. 


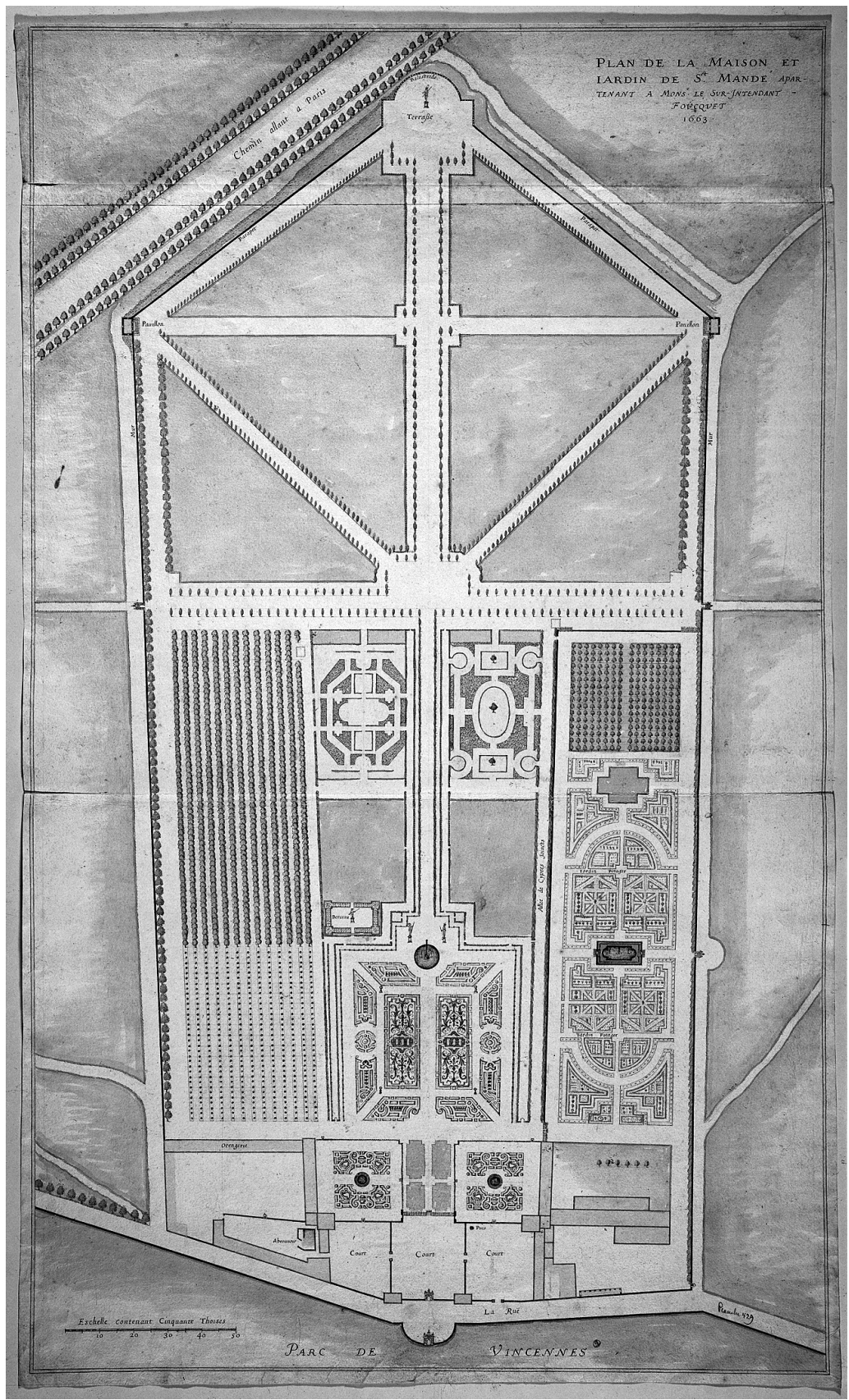

Abb.1: »Plan de la maison et jardin de St. Mandé« (1663). Nationalmuseum Stockholm, Sammlung Cronstett (THC 429). 
ein Gesamtplan der Anlage aus dem Stockholmer Nationalmuseum, der in seiner Gliederung eine wenig strukturierte Gebäudeanlage zeigt.

Fouquet ließ den vorhandenen Baubestand bestehen und veränderte, vergrößerte und ergänzte die einzelnen Gebäude nach seinen Vorstellungen. So entstand eine Anlage mit sechs Innenhöfen und einem großen Park ${ }^{11}$. Der Stockholmer Plan zeigt mehrere Gebäudeteile, die sich im östlichen Teil der Anlage zum Garten von Vincennes hin befinden, unter ihnen die im Südosten gelegene Orangerie sowie die Menagerie, deren »cour [...] est la cinquieme des grandes cours de laditte maison, je dis la cinquieme, et non la derniere, d'autant que toutes les cours sont sous une meme ligne en face de la grande et principalle porte de l'entrée de laditte maison «12. Die einzelnen Gebäudeteile waren durch drei unterirdische Gänge miteinander verbunden, denn »l'esprit, et l'aplication dudit Sieur foucquet [sic] dans ses batiments etoient d'y pouvoir cheminer sous terre, sans etre veu «13. Für die Arbeiten verpflichtete Fouquet begehrte Künstler: Kein Geringerer als Gianlorenzo Bernini, der später Entwürfe für die Ostfassade des Louvre lieferte, war an der architektonischen Umgestaltung beteiligt, ebenso Louis Le Vau, der Erbauer von Vaux-le-Vicomte $^{14}$. Der maître maçon Patel, der ebenfalls für den König am Louvre tätig gewesen war, koordinierte die Bauarbeiten in Saint-Mandé und erhielt von Fouquet die Auflage,

que de faire des bâtimens bas et à un seul étage de crainte que l'elevation n'eu déplut a sa Majesté, qu'a cette fin tous le coté de la couverture desdits batiments que l'on voit de vincennes n'etoit couvert, et ne se devoit couvrir que de Thuiles, et l'autre coté d'ardoises, de

11 Die Anlegung des Gartens erforderte zahlreiche Veränderungen der Landschaft, wie zum Beispiel der Zuschüttung eines kleinen Tales - Arbeiten, die an die aufwendigen landschaftlichen Umgestaltungsmaßnahmen von Vaux erinnern.

12 Discours sommaire de ce qui s'est passé, et a été inventorié de plus remarquable a St. Mandé, BN ms. fr. 10958, fol. 231r.

13 Zuvor heißt es: »Le Masson [=Patel] qui conduit le batiment de cette maison, et qui conduit aussi celui du Louvre, interrogé par serment, s'il scavoit qu'il y eut icy quelque cache, nous a déclaré n'en scavoir point, et ne croit pas qu'il y en eut qu'une, qui n'etoit que commencée, a laquelle il nous a mené, et dans laquelle nous n'avons rien trouvé, n'etant pas encore fermée, elle est dans l'epaisseur de la voute du troisieme chemin sous terre «. (Discours sommaire de ce qui s'est passé, et a été inventorié de plus remarquable a St. Mandé, BN ms. fr. 10958, fol. 231v.) Aus der Verteidigungsschrift von FouQuet, Euvres, Bd.IV, S.159f. wissen wir, dass einer der drei Gänge die beiden von Fouquet als Arbeitszimmer genutzten cabinets miteinander verband.

14 Bernini besuchte Frankreich erst im Jahr 1665. Die Pläne für Saint-Mandé muss er also von Rom nach Paris geschickt haben. Im »Journal de voyage du cavalier Bernin de France« findet sich folgende, von Paul Fréart de Chantelou paraphrasierte Passage: »Il a dit que, dans tous les ouvrages qu'il a faits en France, rien ne s'est démenti, au palais Mazarin, à Saint-Mandé et ailleurs, et qu'il se servait de mortier de chaux éteinte à l'italienne avec sable de rivière et autre du lieu « (Eintrag vom 17. September 1665, in: Fréart de Chantelou, Journal du voyage, S.186). Zu Louis Le Vau in Saint-Mandé siehe: FeLKaY, »Le Vau«, in: DGS, S. 870. 
sorte que venant dudit lieu de vincennes, l'on ne pense voir que >vilia tuguria< et du coté de Conflans on croit voir une pompeuse villa ${ }^{15}$.

Der Bau sollte sich in seiner äußeren Architektur bescheiden zeigen und sich dem nahegelegenen Vincennes visuell unterordnen. In seiner Innenarchitektur jedoch ließ er eine andere Intention erkennen. Von Fouquets Anklägern wurden die Räume nach 1661 als »si superbe, \& avec tant d'ornemens « bezeichnet »que les sommes qui y sont esté dépensées, excedent celles des plus hautes entreprises ${ }^{16}$. Informationen zur Innenarchitektur sind ebenso rar wie zum Bau selbst. Drei nach der Verhaftung des Finanzministers erstellte Inventare der Büsten, Bücher und Medaillen, die sich in SaintMandé befanden, geben geringe Details der Raumaufteilung und Innenausstattung preis ${ }^{17}$. Im Mittelpunkt des Schlosses befand sich ein Salon mit antiken Statuen Merkurs und Apollons. In der nahegelegenen Galerie war ein großer Teil der Bibliothek und der Kunst- und Kuriositätensammlung untergebracht. Im Esszimmer stand »une fontaine de marbre blanc avec sa coquille et un enffant au-dessous $^{18}$. Darüber hinaus bestand der Bau aus einer Abfolge von Räumen und Appartements. Mit der Ausschmückung der Innenräume wurde der soeben aus Italien zurückgekehrte Charles Le Brun betraut: »M. Fouquet l'employa [...] à Saint-Mandé [...], et on y voit le plafond d'un salon, où M. Le Brun a représenté le Soleil levant avec tous les accompagnements convenables du sujet «19. Das Sonnenthema sollte auch in dem nächsten Schlossbau des Finanzministers eine wichtige Rolle spielen ${ }^{20}$. Mit Le Brun waren in Saint-Mandé Louis Le Brun und François Bellin tätig, die nach seinen Entwürfen Arbeiten ausführten ${ }^{21}$. Für die skulpturale Ausschmückung wurde 1655 Michel Anguier eingestellt, der bis 1658 in Saint-

15 Discours sommaire de ce qui s'est passé, et a été inventorié de plus remarquable a St. Mandé, BN ms. fr. 10958, fol. 232v. Zu Patel im Dienst Fouquets siehe: FouQuet, Euvres, Bd. XII, S.181.

${ }^{16}$ Fouquet, CEuvres, Bd. VI, S. 138.

17 Die »Prisée des bustes étant à Saint-Mandé« (AN O $\left.{ }^{1} 1964\right)$ datiert vom 26. Februar 1666; der »Inventaire, prisée et estimation des livres trouvés à Saint-Mandé, appartenant ci-devant à M. Foucquet« (BN ms. fr. 9438) wurde am 31. Juli 1665 erstellt. Die »Estimation de médailles trouvées chez M. Foucquet« (AN O $\left.{ }^{1} 1964\right)$ stammt vom 6. Mai 1666.

18 Prisée des bustes étant à Saint-Mandé (1666), in: BonnAfFé, Foucquet, S. 63.

19 Guillet de Saint-George, Mémoires, Bd. I, S. 440.

${ }^{20}$ Ein Vergleich der beiden Entwürfe ist aufgrund der geringen Informationen zu der Deckenbemalung in Saint-Mandé nicht möglich.

21 CoRdey, Vaux-le-Vicomte, Dokument XI, S. 232-237: Copies de quittances délivrées par divers artistes à Le Brun pour le paiement de travaux exécutés à Vaux-le-Vicomte; darin: »Quittance de Louis Lebrun du 30 mars 1661 pour 600 l. [...] sur les ouvrages de peinture d'ornements que j'ay faict pour Monsieur le Procureur général tant à sa maison de SaintMandé que celle de Paris «(S.233); »Quittance de F. Bellin du 19 juillet 1661, pour 10001. [...] à bon compte des ouvrages de peinture [...] faict pour Monsieur le Procureur général, tant à Vaux qu'à Saint-Mandé, le tout du dessing et conduitte de mon dit sieur Le Brun « (S. 234). 
Mandé arbeitete 22 . Auch Jacques Sarrazin lieferte Skulpturen für die neue Domäne ${ }^{23}$. Die Möblierung des Hauses lässt sich nicht rekonstruieren. Da Fouquet den größten Teil der Ausstattung anlässlich der königlichen Empfänge im Sommer 1661 von Saint-Mandé in sein neues Schloss Vaux-leVicomte hatte transportieren lassen, war sie nach dem Sturz des Finanzministers äußerst spärlich ${ }^{24}$.

Eindeutigere Aussagen als über die Architektur und die Ausstattung des Gebäudes lassen sich über den Garten von Saint-Mandé machen, der sich gen Westen hin erstreckte. Auf dem Stockholmer Plan von 1663 ist ersichtlich, dass die Anlage bereits eine symmetrische und geometrische Form à la française aufzeigte. Die Konzeption des Gartens wird deshalb André Le Nôtre zugesprochen, der allgemein als Schöpfer des französischen Gartens gilt, zum Zeitpunkt der Anlage von Saint-Mandé aber noch am Beginn seiner Karriere stand. Bei dem schwedischen Plan könnte es sich um einen der ersten Quellenbelege für den neuen Gartentypus handeln. Saint-Mandé würde demnach zu den frühen jardins à la française Le Nôtres zählen. Der berühmte Gartenarchitekt setzte hier bereits wichtige Stilmittel des neuen Gartentyps ein. Die geometrische Anlage erstreckt sich entlang einer Hauptachse. Wie in späteren Gärten Le Nôtres sind die Proportionen mit zunehmendem Abstand von den Gebäuden großzügiger bemessen, auch wenn die Anlage mit einer Länge von nur 110 Metern kleiner dimensioniert war als die Gärten anderer ministerieller Schlösser ${ }^{25}$. Aufgrund dieser gartenbaulichen Neuheit vermutet Brix, dass der Garten von Saint-Mandé gleichzeitig mit der Anlage von Vaux-leVicomte entstand ${ }^{26}$. Für eine Anlage durch Le Nôtre spricht auch die Ähnlichkeit mit späteren Gärten. So kehrt in den Tuilerien, die der Gartenarchi-

22 Guillet de Saint-George, Mémoires, Bd. I, S.440: »En 1655, M. Fouquet, surintendant des finances, employa M. Anguier, pour toute la sculpture qui se voit dans la belle maison de Saint-Mandé«.

${ }^{23}$ Die »Prisée des bustes étant à Saint-Mandé« vom 26.Februar 1666 zählt zu dem Bestand des Magazins von Saint-Mandé »une Venus de Monsieur Sarrazin non achevée « (AN O ${ }^{1}$ 1964, abgedruckt in: Bonnaffé, Foucquet, S. 63). Von Michel werden der Tischlermeister Jean Lepautre und der Stuckateur Pietro Sassi als weitere Künstler genannt (Michel, Vatel, S.38).

${ }^{24}$ In dem Bericht der Inventaristen heißt es daher: »Je ne parle point des meubles et ustancils qui ne sont pas icy fort considerables, nous n'y trouvons ny or, ny argent, n'y pierreries, ny meme de vaisselles d'argent, qu'en tres petit nombre«. Weiter lautet es: »Le surplus ayant été porté à Vaux, lors du grand festin, comme les serviteurs nous disent « (Discours sommaire de ce qui s'est passé, et a été inventorié de plus remarquable a St. Mandé, BN ms. fr. 10958, fol. 229v.).

${ }^{25}$ Die Achse von Vaux-le-Vicomte war über einen, jene von Maisons über fünf Kilometer lang.

26 BRIX, Garten, S. 44. Die Arbeit des Gartenarchitekten für Nicolas Fouquet ist ab 1653 belegt. Ob er zunächst den Garten von Saint-Mandé entwarf und sich in einem zweiten Schritt mit der Anlage von Vaux-le-Vicomte auseinandersetzte oder möglicherweise beide Gärten parallel konzipierte, ist unklar. Siehe hierzu: Kapitel 3, S. 86. 
tekt ab 1663 gestaltete, eine mit den beiden Rasenparterres von Saint-Mandé vergleichbare Ornamentik wieder ${ }^{27}$.

Der exotische und ausgefallene Pflanzenbestand des Gartens von SaintMandé, in dem es »deux cent grands orangers, quelques statues et force plantes de noms [...] inconnus et barbares $\aleph^{28}$ sowie unzählige Tulpen und Anemonen gab, wurde von dem deutschen jardinier Jakob Besseman gepflegt. $\mathrm{Zu}$ der Anlage gehörte zudem ein großer Gemüsegarten, den Charles de La Noué bewirtschaftete ${ }^{29}$. Ein Kräutergarten lieferte Jean Pecquet, dem Hausarzt von Fouquet, die Grundstoffe für seine Medikamente.

Mit Hilfe der Künstler und Gärtner wurde Saint-Mandé zu einer maison de plaisance, die Sir Christopher Wren bei seinem Besuch in Paris mit den größten Schlössern der Zeit in einem Atemzug nannte ${ }^{30}$. Wenngleich die Anlage einer architektonischen Patchworkarbeit glich und sich mit Rücksicht auf das nahegelegene Vincennes in seiner äußerlichen Gestalt eher bescheiden zeigte, wurde das Anwesen den repräsentativen Anforderungen gerecht, die an einen Finanzminister gestellt wurden. Ab 1656 ist der Empfang königlicher Gäste in Saint-Mandé belegt. Am 25. Mai 1656 empfing der Finanzminister »la plus-part de la Cour, en son beau séjour de Mandé« und bewirtete die Gäste mit den exklusivsten Delikatessen: »Les sauces vrayment sans-pareilles, les ragoûts frians et merveilles, les fleurs brillans de tous côtez, les bisques, gâteaux et pâtez, et les tourtes de fines herbes, de ce Banquet des plus superbes « ${ }^{31}$. Es folgte ein Besuch Gastons, des Onkels Ludwigs XIV., der am 13. August desselben Jahres auf der Rückreise von Blois in Saint-Mandé eine Pause einlegte »où l'on vid tourner mainte broche, pour rotir de bon gibier frais. L'afluence, en ce lieu fut grande, tant de fruit que de viande; j'ay sceu, sans aller au Dévin, qu'on y but pour cent francs de vin «32. Am 11. November 1657 gab erstmalig Ludwig selbst, in Begleitung Mazarins, seinem Finanzminister für einige Stunden die Ehre eines Besuches ${ }^{33}$.

Mit Saint-Mandé schuf Fouquet einen Ort, der ihm die Führung der Staatsfinanzen im hierfür gebotenen Rahmen erlaubte und in seiner Anlage und Ausstattung der gesellschaftlichen Stellung eines Finanzministers gerecht

\footnotetext{
27 BRIX, Garten, S. 44.

${ }^{28}$ Mémoire adressé au chancelier Séguier sur Fouquet par le conseiller d'État de la Fosse (6 octobre 1661), Bibliothèque imp. Saint-Germain, ms. fr. 709, fol. 145; zitiert nach: CHÉRUEL, Mémoires, Bd. I, S. 487.

${ }^{29}$ Fouquet, Euvres, Bd.IV, S.180: »Jacques Bessemen, Hambourgeois de nation, Jardinier fleuriste, \& ayant charge des Fontaines; Charles de la Noüé, Jardinier Potager; \& Perrine Tarton sa femme, ayant soin de la Conciergerie«.

30 »After the incomparable Villas of Vaux and Maisons, I shall but name Rueil, Courances, Chilly, Essoane, St. Maur, St. Mandé, Issy, Meudon, Raincy, Chantilly, Verneuil, Lioncour, all which, and I might add many others, I have survey'd « (Wren, Parentalia, S.261f.).

31 Muze historique, Bd. II, Lettre XXI, 27 may 1656, S. 199, Vers 213-236.

32 Ibid., Lettre XXXIII, 19 août 1656, S.213, Vers 22-28.

${ }^{33}$ Eine kurze Beschreibung dieses Besuchs findet sich in: ibid., Lettre XLV, 17 novembre 1657, S. 405.
} 
wurde. Die unmittelbare Nähe zu seinem politischen Mentor und Förderer Mazarin zeigt die enge Bindung, die Fouquet auch weiterhin an den Premierminister erhalten wissen wollte. Nicolas, der seine Karriere der Protektion der beiden Premierminister zu verdanken hatte, machte mit der Wahl des Standorts Saint-Mandé zudem seine Hoffnung auf eine weitere Protegierung durch Mazarin deutlich.

Saint-Mandé war jedoch nur zu einem Teil den beruflichen Verpflichtungen des Finanzministers geschuldet. Es wurde zudem zum bevorzugten Aufenthaltsort Fouquets und seiner Familie. Die repräsentativen Innenräume waren mit ihren vielen Appartements und den unterirdischen Gängen auf die Bedürfnisse der Bewohner abgestimmt. Das neue Anwesen war ein Bau, der sowohl den Anforderungen und Verpflichtungen des Finanzministers als auch des Privatmanns Fouquet gerecht wurde.

\section{Bibliothek, Kuriositätensammlung und Kunsterwerb}

Die Betätigung als Sammler wurde von dem neuen Finanzminister ebenso erwartet wie die Errichtung einer repräsentativen Schlossanlage. Auch Sammlungen waren ein Mittel der gesellschaftlichen Distinktion: »les collections sont pour les détenteurs du pouvoir des insignes de leur supériorité «34. Gerade für einen Aufsteiger wie Fouquet war es wichtig, sich mit allen verfügbaren Mitteln zu legitimieren. Persönliche Vorlieben spielten dabei nur eine untergeordnete Rolle.

Trotzdem können diese nicht unbedacht bleiben. Gerade in der ersten Phase der Kulturpatronage des Finanzministers konnte man an seinen Sammlungen die Neigungen seiner Eltern erkennen. Im Falle der Bücher-, Karten- und Medaillensammlung baute Fouquet sogar direkt auf dem Erbe des Vaters auf, der seine Sammlungen ebenfalls als ein »billet d'entrée dans un milieu fermé «35 genutzt hatte. Für den Finanzminister galt nun, diese dem weiteren Aufstieg der Familie anzupassen ${ }^{36}$.

\footnotetext{
34 Pomian, Collectionneurs, S. 53.
}

35 Ibid.

${ }^{36}$ Aufgrund der großen Ungenauigkeit der raren Quellen sowie der nicht immer klar vorzunehmenden Abgrenzung der zwei Phasen der Kulturpatronage Fouquets ist die Zuordnung der Neuanschaffungen der einzelnen Sammelgebiete nicht immer eindeutig vorzunehmen. Dies gilt vor allem für die Kuriositätensammlung. Daher finden sich auch Käufe, die erst nach 1655 (also in der zweiten Phase der Kulturpatronage) getätigt wurden, jedoch stark der Tradition der ersten Sammelphase verpflichtet sind, bereits in diesem Kapitel. Auch die Analyse des Bibliotheksbestandes, der sich erst nach 1655 stark vergröBerte, findet sich in diesem Kapitel, da die Buchsammlung nicht nach Vaux-le-Vicomte umzog, sondern in Saint-Mandé verblieb. 


\subsection{Die Bibliothek}

Die Erfindung der Buchdruckerkunst in der Alten Welt sowie die Verbreitung der gedruckten Bücher zu Beginn des 17. Jahrhunderts übten in Europa einen nachhaltigen Einfluss auf die Entwicklung des Bibliothekswesens aus. Im Grand Siècle waren private Büchersammlungen unter den französischen Staatsmännern und Financiers weit verbreitet. Wie alle Arten von Sammlungen gehörte auch die Bibliothek zu einem Mittel gesellschaftlicher Abhebung, denn sie bedeutete nicht allein die Aufwendung großer Geldmittel und damit die Zurschaustellung von Wohlstand. Sie galt zugleich als ein Ort der Wissenschafts- und Literaturpatronage, die dem Förderer Ansehen über die Grenzen seiner Stadt hinaus einbrachte. Voraussetzung für den Aufbau einer imponierenden Bibliothek war neben beträchtlichen finanziellen Mitteln ein großes Netzwerk an Klienten in ganz Europa, das die Anschaffung wertvoller Manuskripte und seltener Drucke ermöglichte. Fouquets Sammlungen wiesen einen großen Bestand an griechischen und lateinischen Editionen, aber auch an spanischer, italienischer, englischer und deutscher Literatur und an handschriftlichen Raritäten aus dem Orient auf, die nur mit Hilfe von Klienten im Ausland beschafft worden sein konnten. Die berühmtesten Bibliotheken im Land besaßen im 17. Jahrhundert die Männer, die dank ihres Amtes sowohl über finanzielle Ressourcen als auch über ein weites Netzwerk im Ausland verfügten: die Premierminister Richelieu und Mazarin ${ }^{37}$. Die Größe ihrer Büchersammlungen wird im Jahr 1643 auf 6380 im Falle Richelieus und im Jahr 1648 auf 40000 im Falle Mazarins geschätzt ${ }^{38}$. Darüber hinaus ist die Bibliothek des Kanzlers Séguier zu nennen, die mit einem Bestand von 30000 gedruckten Büchern im Jahre 1672 mit der Buchsammlung Mazarins vergleichbar ist ${ }^{39}$. $\mathrm{Zu}$ den Bibliotheken gehörte indes nicht allein ein großer Buchbestand. Auch Medaillen, Karten, Skulpturen und Kuriositäten aller Art waren in ihren Räumen untergebracht, die als Orte der Wissenschaft und wissenschaftlicher Zusammenkünfte dienten.

Die neue Bibliothekskultur beeinflusste die Achitektur des 17. Jahrhunderts. Galerien wurden zu bevorzugten Heimstätten der kostbaren Buchbestände, die meist noch weitere Räumlichkeiten in den Stadt- und Landhäusern beanspruchten. Die großen Bestände wurden von Bibliothekaren verwaltet, die sich im Laufe des Jahrhunderts zu einem eigenen Berufsstand

37 Martin, Livre, Bd.I, S. 477 gibt an, dass in der Mitte des 17. Jahrhunderts circa siebzig Privatbibliotheken mit einem Bestand von 3000 bis 4000 Büchern in Frankreich existierten: »Parmi ceux-ci, six sont des princes du sang ou des ministres; quatre des conseillers d'État; cinq des maîtres des requêtes; huit des présidents de cours souveraines; sept des officiers de ces cours; neuf des avocats; deux des financiers, et une vingtaine des théologiens ou des érudits dont sept médecins «. Die berühmteste unter ihnen war ohne Zweifel lange Zeit die Bibliothek des Pariser Parlamentspräsidenten Jacques-Auguste de Thou.

38 JoLLY, Bibliothèques françaises, S. 127, 135.

39 Ibid., S. 151. 
herausbildeten. Sie katalogisierten die Bücher und entwickelten neue Klassifizierungsmethoden ${ }^{40}$.

Seitdem Nicolas Fouquet im Jahr 1640 die 15000 Bände umfassende Bibliothek seines Vaters geerbt hatte, wurde diese von seinem ehemaligen Lehrer am Collège Clermont, dem Jesuitenvater Deschampsneufs betreut ${ }^{41}$. Obwohl er über ein eigenes Appartement in Saint-Mandé verfügte, scheint Deschampsneufs nur einen Teil seiner Arbeitskraft der Bibliothek gewidmet zu haben; in einem Brief an Chamillart vom 17. Juni 1655 beschwert er sich über den Mangel an Zeit, der es ihm nicht erlaube, eine neue Klassifikation der Werke einzuführen $^{42}$. Seine Arbeit beschränkte sich vielmehr auf die Aufnahme der Neuzugänge in einem alphabetischen Katalog. Inwieweit er auch bei dem Erwerb neuer Bücher behilflich war, bleibt ungewiss.

Bis 1655 wurde die väterliche Bibliothek kaum vermehrt. Nur wenige Quellen berichten von Erwerbungen während der ersten fünfzehn Jahre des Bestehens der Bibliothek Nicolas Fouquets. Es ist anzunehmen, dass der Finanzminister, welcher in den vierziger und fünfziger Jahren ein fleißiger Salonbesucher war und auch selbst zur Feder griff, bevorzugt Werke seiner Lieblingsdichter in die Bibliothek aufnahm ${ }^{43}$.

Ab 1655 begann Nicolas Fouquet mit dem systematischen Ausbau seiner Büchersammlung. Die Vergrößerung des Bibliotheksbestandes wurde durch eine veränderte Anschaffungsmethodik ermöglicht, die den 1627 mit seiner »Advis pour dresser une bibliothèque« veröffentlichten Ratschlägen Naudés folgte. In seinem Werk empfiehlt Naudé als schnellste und leichteste Möglichkeit zur Vergrößerung des eigenen Buchbestandes den Erwerb einer kompletten fremden Bibliothek. Der Aufkauf erlaube es, an einem Tag zu erwerben, was im Normalfall ein ganzes Menschenleben erfordere ${ }^{44} .1655$ kaufte Fouquet die Bibliothek des vier Jahre zuvor verstorbenen Erzbischofs von Toulouse, Charles de Montchal, der vorzugsweise griechische und lateinische

${ }^{40}$ Mit seiner »Advis pour dresser une bibliothèque« wurde einer von ihnen, der spätere Bibliothekar Mazarins Gabriel Naudé, 1627 zum Begründer der modernen Bibliothekswissenschaft.

${ }^{41}$ Guy Patin spricht in einem Brief an Charles Spon aus dem Jahre 1657 gar von zwei Jesuiten, die sich in Saint-Mandé um den großen Buchbestand kümmerten: Patin, Lettres, Bd.II, S. 276 in einem Brief an Charles Spon vom 16. Februar 1657, in dem er den Kauf medizinischer Bücher aus der Bibliothek des verstorbenen Moreau schildert: »Deux jésuites y [à Saint-Mandé] sont qui les [les livres de médecine] arrangent «.

42 BN ms. lat. 17172, fol. $95 \mathrm{r} .-98 \mathrm{v}$.

43 Saunders, Politics, S.6. Ein Teil der im Inventar der Fouquetschen Bibliothek aufgeführten Werke lassen über ex libris ihre Herkunft aus der Bibliothek von Léonor d'Estampes de Valençay erkennen. Da nur ein geringer Bestand der Büchersammlung von Estampes de Valençay in den Besitz Fouquets gelangte, kann man davon ausgehen, dass der Finanzminister nur einzelne Werke erwarb und es sich bei dem Kauf um keinen Erwerb der kompletten Bibliothek handelte.

${ }^{44}$ NAUdé, Advis, S. 68. 
Autoren gesammelt hatte ${ }^{45}$. Ein Jahr später erfolgte der Aufkauf der Bibliothek des Mediziners René Moreau ${ }^{46}$. Fouquet selbst berichtet in seiner Verteidigungsschrift von weiteren Bibliotheksaufkäufen namhafter Sammler: »La bibliothèque m'a esté donnée par mon Pere, j'y ay fait quelque augmentation, \& les Livres de MM. de Morangis, le Ragois, Clement, Arnoul, Cramoisy \& autres, sont encore dûs, sans compter ce qui m'a esté donné par les Auteurs \& Libraires tous les ans ${ }^{47}$. Cordey erwähnt darüber hinaus den Aufkauf der Bibliothek Harlays und jener Trichet du Fresnes, dem Fouquet seinen reichen Bestand an Literatur zur italienischen Geschichte zu verdanken hatte ${ }^{48}$.

Wie Fouquet in seiner Verteidigungsschrift berichtet, wurden die Käufe durch Schenkungen der ihm und seiner Familie gewidmeten Werke ergänzt. Diese nahmen nach 1657 mit der Einstellung Paul Pellissons und der damit einhergehenden institutionalisierten Literaturförderung durch den Finanzminister erheblich zu. Die Vielschichtigkeit seines Bestandes lässt darüber hinaus vermuten, dass Fouquet ein breites Netzwerk für den Erwerb von Büchern und Handschriften in ganz Europa nutzte. Seine Stellung ließ ihn in direktem Kontakt mit Botschaftern und anderen Gesandten stehen, die nicht nur diplomatische Missionen zu ihren Aufgaben zählten. Auch das Aufspüren und der Erwerb von Büchern, Kunstobjekten und Kuriositäten sowie die Korrespondenz mit ihren Pariser Patronen gehörte zu ihrem Arbeitsalltag ${ }^{49}$. Aus seinem weiten Netz an Klienten und Financiers in Frankreich selbst mögen ebenso Schenkungen an die Bibliothek des Finanzministers gegangen sein. Bekannt sind vier Agenten, die von Fouquet für Einkäufe ins europäische Ausland geschickt wurden. Bei drei von ihnen kann jedoch nur vermutet werden, dass sie als Buchkäufer tätig waren. Louis Fouquet, der 1655 im Auftrag seines Bruders für Kunst- und Raritätenkäufe nach Rom reiste, erwähnt in den Briefen an seinen Bruder die Übersendung von Architekturdarstellungen und -abhandlungen ${ }^{50}$. Es ist nicht auszuschließen, dass er noch weitere Einkäufe für die Bibliothek tätigte. Auch der graveur Bertinetti unternahm wiederholt Reisen im Auftrag seines Patrons »pour aller traiter des négociations à l'étranger «51. Über die genauen Daten und Abläufe seiner Auslandsaufent-

45 Die Sammlung umfasste »trois cent six volumes latins, cent onze grecs, quatorze hébreux, cinquantetrois françois et seize italiens « (PAULIN, Manuscrits françois, S. 23).

46 Brief Guy Patins an Charles Spon vom 16. Februar 1657, in: PAтIN, Lettres, Bd. II, S. $275 f$.

${ }^{47}$ Fouquet, Euvres, Bd. VI, S.139. Fouquet kaufte zudem die Bibliothek von Harlay und einen Großteil der Manuskripte aus der Bibliothek von Montchal.

48 Cordey, Bibliothèque, S.10, 12. Siehe auch: BN ms.lat.17172, fol.66-93: Livres de Mr du Fresne [...] pris a St. Mandé.

49 Perrin, Netzwerk, S. 186.

${ }^{50}$ Brief Louis Fouquets an seinen Bruder vom 2. August 1655, abgedruckt in: FouQueT, Lettres, S. 290.

51 GRÉsy, Graveur, S.101. Grésy gibt in seinem Aufsatz zu Bertinetti das Leben des Medailleurs nach einem Manuskript von 1707 mit dem Titel »Heureux chanoine de Rome, nouvelle galante, ou la Résurrection prédestinée, par C.M.D.R., avocat en la cour« (1707) wieder. 
halte ist nichts überliefert. Vermutlich versorgte der Italiener den Minister im Zuge seiner Reisen mit Druckerzeugnissen. Ebenso lässt sich auch hinsichtlich der römischen Kunstmission von François Maucroix des Jahres 1661 nur vermuten, dass sie Buchkäufe einschloss. Im gleichen Jahr reiste der Bibliothekar Carcavy im Auftrag des Finanzministers nach Italien ${ }^{52}$. Obgleich über den Sinn und Zweck der Reise nichts bekannt ist, kann es in seinem Fall als sicher gelten, dass dieser sich hier um den Erwerb von Büchern und Handschriften bemühte.

Nicht nur in der Art des Aufbaus und Ausbaus der Bibliothek kann man bei Fouquet eine Orientierung an Naudé feststellen. Auch mit der Öffnung der Bibliothek für das Publikum folgte der Finanzminister den Empfehlungen des berühmten Bibliothekars, dem zufolge »es kein ehrenvolleres \& sichereres Mittel gibt, sich ein großes Ansehen unter den Volksmassen zu verschaffen, als schöne \& prächtige Bibliotheken zu errichten, um sie dann der Öffentlichkeit zur Benutzung zu weihen \& zu widmen $\ll^{53}$. Dem Vorbild seines politischen Mentors Mazarin folgend machte er ab 1657 seine Bibliothek Gelehrten zugänglich ${ }^{54}$. Den zahlreichen Dichtern, die in Saint-Mandé auf eine Unterredung mit dem Hausherrn hofften, wurde sie darüber hinaus zu einem angenehmen Warteraum: »C'est dans les bibliothèques qu'on attend ces précieux moments qu'il dérobe aux occupations qui l'accablent « ${ }^{55}$.

Mit dem Anwachsen des Buchbestandes war eine neue und intensivere Form der Betreuung notwendig geworden. Der Finanzminister nahm daher 1658 Pierre de Carcavy in seine Dienste. Der ehemalige Magistrat und Gelehrte hatte vor dem finanziellen Ruin seiner Familie im Jahre 1648 selbst eine bedeutende Bibliothek besessen und widmete sich seit Beginn der fünfziger Jahre der »recherche des livres rares qu'il revendoit aux curieux lorsqu'il y trouvoit son compte $\ll^{56}$. Seine reichen Kontakte mit Gelehrten in ganz Europa machten ihn zu einer unersetzlichen Figur beim Aufbau einer wissenschaftlichen Bibliothek. Über neueste Publikationen war er stets aufs Beste informiert. Seine enge Beziehung zu dem Mathematiker, Physiker und Astronom Huygens, mit dem er einen regen Briefkontakt pflegte, und dem Mathematiker Fermat, hauptsächliches Sujet des Briefwechsels zwischen Huygens und Carcavy, begünstigte die Anschaffung der neuesten Literatur auf dem Gebiet der Mathematik.

Über die Aufgabenverteilung zwischen Carcavy und Deschampsneufs, der weiterhin in den Diensten des Finanzministers stand, ist wenig bekannt. Mög-

52 Chatelain, Foucquet, S.311f.

53 Naudé, Advis, S.20f.

54 »M. Fouquet veut faire une bibliothèque publique de ses livres«, in: Patin, Correspondance, Brief an Ch. Spon vom 13. März 1657, S. 207.

55 Corneille, Edipe, Au Lecteur, in: Conneille, CEuvres, S.124. Auch La Fontaine berichtet in einer Epître von einer Wartezeit in der Bibliothek (LA FontaInE, CEuvres complètes II, Bd. II, S. 502).

56 BN ms. fr. n. a. 1327, fol. 218. 
licherweise war der Jesuitenvater, der nach dem Fall Fouquets ein Inventar der Bibliothek erstellte ${ }^{57}$, für die Katalogisierung und Carcavy für die Erweiterung des Bestandes zuständig.

Der Bestand der Bibliothek von Saint-Mandé erfasste nach Angaben Deschampneufs im Jahr 1661 rund 27000 Bände, davon 7000 in-folio, zwischen 7000 und 8000 in-quarto, rund 12000 in-octavo (oder kleiner) und um die 1050 Handschriften ${ }^{58}$. Als im Jahre 1665 anlässlich des Verkaufs der Fouquetschen Besitzungen ein weiteres Inventar der Bibliothek erstellt wurde, war der Bestand auf weniger als 20000 Bände gesunken, obwohl die Bibliothek in den vier Jahren zwischen 1661 und 1665 versiegelt gewesen war. Deschampsneufs führt den Verlust auf Diebstähle zurück, da es trotz der Versiegelung mannigfache Zutrittsmöglichkeiten gegeben hatte ${ }^{59}$. Bei dem 1661 von Deschampsneufs erstellten Katalog handelt es sich um kein vollständiges Inventar, das eine Rekonstruktion des Buchbestandes erlauben würde. Auch eine Analyse des Bestandes auf Grundlage des Inventars von 1665 gibt wegen der Einbrüche und Entwendungen kein ganz sicheres Bild der Bibliothek wieder ${ }^{60}$. Sie liefert jedoch wichtige Aufschlüsse über den Standort der Bücher und die bevorzugten Sammelgebiete des Finanzministers.

In Saint-Mandé folgte Fouquet den neuen architektonischen Vorgaben seines Jahrhunderts: Der Großteil der Bücher war in der Galerie untergebracht, die in dem nördlich an die Orangerie angrenzenden Bau entstanden war ${ }^{61}$. Hier befand sich eine Sammlung der Heiligen Schriften in vielen Übersetzungen, darunter »la Biblia regia de Platin, en huit volumes, [...] la Biblia graeca, d'Alde Manuce [...] des bibles de Robert Estienne, Simon de Colines, de l'Imprimerie royale, et surtout un exemplaire de la célèbre Bible imprimée à Mayence par Fust et Schoiffer, en $1462 \ll^{62}$. Die Bibeln fanden Ergänzung

57 BN ms. lat. 17 172: Extraict de l'inventaire fait à Saint-Mandé en 1661.

58 Ibid.

59 Deschampsneufs in einem Brief an Chamillart vom 17. Juni 1665, BN ms. lat.17172, fol. 95r.-98v. Vor allem der Bestand an kleinformatigen Büchern, die sich gut in der Kleidung verbergen lassen, war erheblich geschrumpft.

${ }^{60} \mathrm{BN}$ ms. fr. 9438. 1667 wurde anlässlich des anstehenden Verkaufs der Bibliothek von Saint-Mandé zudem bei Denis Thierry in Paris ein »Mémoire des manuscrits de la bibliothèque de M. Fouquet« herausgegeben (BN Impr. 7975).

61 In dem »Inventaire, Prisée \& Estimation des livres trouvés à St. Mandé« von 1665 (BN ms. fr. 9438) heißt es auf fol. 52 über die Werke lateinischer und gallischer Dichter, sie befänden sich »dans le fon de la gallerie \& du coté de lorangerie soubs les tables des fenetres«. Dies könnte auch darauf hindeuten, dass sich die Galerie in dem Gebäudeteil gegenüber der Orangerie, die als einziges Gebäude auf dem Stockholmer Plan definiert ist, befunden hat. In jedem Fall ist sie dem südlichen Gebäudekomplex der Anlage zuzuschreiben. Neben der Galerie führt die »Prisée des bustes étant à Saint-Mandé« einen Raum mit der Bezeichnung la bibliotheque auf. Unklar bleibt, ob es sich hierbei um einen der drei auf die Galerie folgenden Räume handelt oder um einen im Inventar der Bibliothek nicht aufgeführten Raum (Prisée des bustes étant à Saint-Mandé [26 février 1666], AN O ${ }^{1}$ 1964, abgedruckt in: BonNAFFÉ, Foucquet, S. 63).

62 Cordey, Bibliothèque, S. 7 . 
durch Kommentare, Bullen, Konzilsbeschlüsse, griechische und lateinische Ausgaben der Kirchenväter sowie Werke zu diversen Ordensgeschichten und zur Kirchengeschichte. Zudem befand sich in der Galerie Fouquets eine Sammlung geographischer und historischer Werke. Selbst der kleinste Raum diente der Unterbringung von Büchern und Papieren: »dans le fon de la gallerie $\&$ du coté de lorangerie soubs les tables des fenetres ${ }^{63}$ fanden sich Manuskripte, Stiche und Nachschlagewerke aller Art.

Drei Räume, die ebenfalls Bestände der Bibliothek aufnahmen, folgten auf die Galerie. Der erste von ihnen barg überwiegend Werke zu Theologie, Philosophie und Mathematik ${ }^{64}$. Der zweite Raum, im Inventar als chambre des Manuscrits bezeichnet ${ }^{65}$, beherbergte die als manuscrits rescens verzeichneten Handschriften aktueller politischer Ereignisse. Im dritten Raum, der Chambre civile $^{66}$, waren juristische Abhandlungen zum Zivil- und Kirchenrecht untergebracht. Von ihm führte eine Treppe zu einer Kammer, in der weitere Handschriften lagerten. Hier fanden sich Schriften von Rabbinern und alten arabischen und hebräischen Gelehrten sowie Ausgaben des Talmud und des Koran. Weitere Manuskripte sowie Werke zu Themen der Theologie und Geographie fanden sich in einem als Bibliothek bezeichneten Raum ${ }^{67}$. Das Inventar verzeichnet zusätzliche Buchbestände in der chambre de la Ménagerie, in der sogenannten apothicairerie, dem Labor von Saint-Mandé, in dem Zimmer und cabinet Mme Fouquets sowie in dem Raum, den der Jesuitenvater Deschampsneufs bewohnte 68 .

Der größte Teil der Bücher war in fahlrotes Kalbsleder eingebunden und trug auf der Vorderseite das Wappen Fouquets, ein nach links kletterndes Eichhorn unter der couronne de vicomte, auf der Rückseite das bibliophile Zeichen Fouquets, ein doppeltes $\Phi^{69}$. Ein kleinerer Teil des Bestandes war in rotes, mit goldenen Randstreifen und Schriftzügen versehenes Ziegenleder (Saffian) eingebunden.

Das Inventar von 1665 führt lediglich die in-folio-Bände und einen geringen Teil der in-quarto-Bände mit ihren Titeln auf. Der Rest wird in Paketen zusammengefasst, die allenfalls einem Fachgebiet zugeordnet wurden. Obwohl es sich bei den mit ihrem Titel aufgezeichneten Werken um weniger als ein Drittel des

63 BN ms. fr. 9438 , fol. 52.

64 Ibid., fol. 59-70.

65 Ibid., fol. 85. Buchbestände des zweiten Raumes aufgeführt ibid., fol. 71-86.

66 Ibid., fol. 87-98.

67 Ibid., fol. 119-144.

68 In der chambre de la Ménagerie waren Werke zur europäischen Geschichte (fol.99-108), in der apothicairerie Bücher über Anatomie und Pflanzenkunde (fol.109), im Appartement von Mme Fouquet Bibeln und geistliche Literatur (fol. 110) und in den Räumen des Père Deschampsneufs Bibeln, Werke zur französischen, arabischen und chinesischen Geschichte und kunsthistorische Bände (fol. 173f.) untergebracht (ibid.).

69 Konsultiert man heute Bände der Bibliothèque nationale oder anderer Bibliotheken in Frankreich aus der Zeit um und vor 1661, so kommt es nicht selten vor, dass man einen Band aus Fouquets Sammlung in die Hände bekommt. 


\begin{tabular}{|c|c|c|c|c|}
\hline \multirow[t]{2}{*}{ Fachgebiet } & \multirow{2}{*}{$\begin{array}{l}\text { Anzahl } \\
\text { der Titel }\end{array}$} & \multicolumn{3}{|c|}{ Vergleichswerte } \\
\hline & & Séguier & Richelieu & $\begin{array}{l}\text { Frz. Produktion } \\
(1641-1645)\end{array}$ \\
\hline \multicolumn{5}{|l|}{ I. Religion } \\
\hline Bibeln & 74 & & & \\
\hline Bibelkommentare & 127 & & & \\
\hline Konzilien & 31 & & & \\
\hline Bullen & 9 & & & \\
\hline Griechische Kirchenväter & 54 & & & \\
\hline Lateinische Kirchenväter & 70 & & & \\
\hline Kirchengeschichte & 90 & & & \\
\hline Ordensgeschichte & 80 & & & \\
\hline Theologie & 62 & & & \\
\hline Kontroverse Literatur & 18 & & & \\
\hline Häretische Literatur & 78 & & & \\
\hline Andere & 18 & & & \\
\hline Gesamt & $711(17,7 \%)$ & $31 \%$ & $30,4 \%$ & $39,5 \%$ \\
\hline \multicolumn{5}{|l|}{ II. Geschichte } \\
\hline Geographie & 102 & & & \\
\hline Chronologien & 436 & & & \\
\hline Griechische Geschichte & 91 & & & \\
\hline Italienische Geschichte & 254 & & & \\
\hline Französische Geschichte & 249 & & & \\
\hline Deutsche Geschichte & 172 & & & \\
\hline Belgische Geschichte & 91 & & & \\
\hline Spanische Geschichte & 146 & & & \\
\hline Britische Geschichte & 33 & & & \\
\hline Türkische Geschichte & 67 & & & \\
\hline Geschichte Asiens & 30 & & & \\
\hline Gesamt & $1671(41,6 \%)$ & $14 \%$ & $25,3 \%$ & $25,2 \%$ \\
\hline \multicolumn{5}{|l|}{ III. Literatur } \\
\hline Griechische Autoren & 50 & & & \\
\hline Lateinische Autoren & 54 & & & \\
\hline Französische Autoren & 15 & & & \\
\hline Humanisten & 49 & & & \\
\hline Wörterbücher & 239 & & & \\
\hline Gesamt & $407(10,1 \%)$ & $18 \%$ & $19,6 \%$ & $22,6 \%$ \\
\hline \multicolumn{5}{|l|}{ IV. Wissenschaft/Philosophie } \\
\hline Medizin & 273 & & & \\
\hline Anatomie & 24 & & & \\
\hline Naturgeschichte & 102 & & & \\
\hline Mathematik & 336 & & & \\
\hline Philosophie & 142 & & & \\
\hline Gesamt & $877(21,8 \%)$ & $15 \%$ & $9,4 \%$ & $7,6 \%$ \\
\hline \multicolumn{5}{|l|}{ V. Recht } \\
\hline Kanonisches Recht & 98 & & & \\
\hline Zivilrecht & 108 & & & \\
\hline Gewohnheitsrecht & 23 & & & \\
\hline Königliches Recht & 125 & & & \\
\hline Gesamt & $354(8,8 \%)$ & $8 \%$ & $4,1 \%$ & $5,1 \%$ \\
\hline Insgesamt & 4020 & & & \\
\hline
\end{tabular}


Tabelle 1: Buchbestand von Saint-Mandé. Verteilung des im Inventar von 1665 (BN ms. fr. 4938) mit Titeln aufgeführten Buchbestandes der Bibliothek von Nicolas Fouquet nach Fachgruppen geordnet sowie die Vergleichswerte der Bibliotheken Séguiers und Richelieus in Prozent (unter Zuhilfenahme von: SAUNDERS, Politics, S. 9f.; NEXON, Bibliothèque, S.151). Anmerkung zu den Vergleichswerten: Die von Nexon unter »Diverses " gemachten Angaben (Séguier: 14\%; Richelieu: 10,7\%) werden hier nicht aufgeführt, so dass die unter Séguier und Richelieu angegebenen Werte keine 100\% ergeben.

Gesamtbestandes handelt, vermittelt die Auswertung der aufgeführten Werke einen Eindruck von den Sammelschwerpunkten (siehe Tabelle 1).

Der Schwerpunkt der Sammlung lag mit rund vierzig Prozent auf dem Gebiet der Geschichte - vornehmlich der französischen und italienischen Geschichte, die, laut Inventar, mit jeweils circa 250 Titeln vertreten waren ${ }^{70}$. Im Vergleich zu den Bibliotheken von Séguier und Richelieu waren dies ungewöhnlich viele. Fouquets Vorliebe für historische Literatur hängt vielleicht mit seinen politischen und gesellschaftlichen Ambitionen zusammen. Denn für seine Karriere war eine profunde historische Bildung von großem Vorteil. Der Bestand an Werken zur europäischen und außereuropäischen Geschichte schloss Abschriften diplomatischer Schriften ein, die sich laut Inventar in der chambre des Manuscrits befanden. Internationale Abkommen, diplomatische Berichte und Korrespondenzen waren hier ebenso zu finden wie Parlamentsregister und Akten großer politischer Prozesse. Insgesamt befanden sich in diesem Raum 460 Manuskripte zum aktuellen Politikgeschehen, die Ergänzung fanden in Stichen großer politischer Ereignisse, königlicher Einzüge sowie herrschaftlicher Feste und Theaterinszenierungen. Für einen aufstrebenden Politiker stellten sie ein ausgezeichnetes Lehrmaterial dar.

Etwas geringer, doch mit mehr als zwanzig Prozent noch immer ungewöhnlich groß im Vergleich zu anderen Bibliotheken, war der Bestand an Werken zu Wissenschaften und Philosophie, der vor allem auf dem Gebiet der Mathematik reich bestückt war. Dieser Schwerpunkt der Sammlung war Carcavys Sachverstand und seinen weitreichenden Beziehungen zu verdanken. Auch der große Bestand an medizinischer Literatur ist erstaunlich. Bedenkt man das starke Interesse Marie de Maupeous, der Mutter Fouquets, an medizinischen Studien sowie die Arbeit Pecquets in Saint-Mandé, mag auch dieser Sammelschwerpunkt nicht erstaunen. Der Bestand konnte durch den Ankauf der Bibliothek René Moreaus um 1656 erheblich erweitert werden. Die Gebiete Theologie und Religionsgeschichte sind mit fast achtzehn Prozent verhältnismäßig schwach vertreten, zumindest im Vergleich zu den Bibliotheken

70 Als die Bibliothek 1667 nach dem Abschluss des Prozesses gegen Fouquet öffentlich zum Verkauf angeboten wurde, soll Carcavy Cordey zufolge einen weit größeren Bestand an Literatur zur italienischen Geschichte für die königliche Bibliothek gekauft haben: »Un certain nombre de manuscrits échut à Le Tellier, archevesque de Reims. Carcavy, devenu bibliothécaire de Colbert, puis du roi, choisit 729 in-folio, 601 in-quarto et 25 paquets d'in-octavio, relatifs à l'histoire d'Italie« (CORDEY, Bibliothèque, S.11f.). 
von Séguier oder Richelieu. Da in den oben angegebenen Zahlen weder die kleinformatige Literatur noch die Manuskripte berücksichtigt sind, kann kein endgültiges Urteil gefällt werden. Ähnliches gilt für den Bereich der Literatur, die mit ihren rund zehn Prozent angesichts der breiten Literaturförderung durch den Finanzminister schwach vertreten scheint. Nahezu sämtliche Werke der von Fouquet geförderten Literaten und der ihm gewidmeten Bücher sind im Inventar nicht mit ihrem Titel aufgeführt. Auch dies mag der Methode der Inventaristen geschuldet sein, die die kleinformatigen Werke in Pakete zusammenfassten. Die juristische Literatur liegt mit 8,8\% nur knapp über dem Bestand der Bibliothek Séguiers. Der Umfang der Sammlung an Werken zur Jurisprudenz mag den Bedürfnissen eines Ministers entsprochen haben.

Noch während des Prozesses gegen Fouquet wurde die Bibliothek des Finanzministers als »une des plus belles, \& peut-estre la plus chere de celles de l'Europe $\ll^{71}$ gerühmt. Nach seinem Sturz zeigte sich die Exklusivität des Buchbestandes im Oktober 1661 ein letztes Mal, als zwei spanische Franziskanermönche die Bibliothek besuchten:

Deux Cordeliers d'Espagne, personnages de lettres et de prudhommes etans venus voir la bibliotheque, moyenant une lettre en passeport de M. le Tellier, ils se sont principalement arretés dans la Chambre ou sont les alcorants, les talmudes, les Rabins, et quelques vieils interpretes de la Bible, et comme je les ay étés prié de venir diner avec nous, ils m'ont fort civilment et religieusement refusé, et que le Livre quils tenoient et sur lequel ils faisoient des recueils etoit d'un autheur Espagnol, qui avoit interpreté, vocabula Bibliorum, et lequel livre ils n'avoient jamais pus voir en Espagne ${ }^{72}$.

Wenngleich der Bestand einer privaten Bibliothek zu der aus der heutigen Zeit geborenen Vermutung verleitet, sie ließe Rückschlüsse auf die Bildung ihres Besitzers zu, können aufgrund der Buchbestände keine Aussagen über die Kenntnisse Nicolas Fouquets gemacht werden ${ }^{73}$. Vielmehr muss man die Frage nach der Intention des Ausbaus der Bibliothek nach 1655 stellen. Die Sammlung entspricht den Bedürfnissen von Gelehrten, Geistlichen und Literaten. Von diesen (Carcavy und Deschampsneufs) wurde sie erweitert, um sie 1657 der gelehrten Öffentlichkeit zugänglich zu machen. Mit der Öffnung seiner Bibliothek schuf der Finanzminister ein wissenschaftliches Forum und trug zur Förderung von Wissenschaften und Literatur bei. Naudé zufolge war dies eines der besten Mittel, um »sich großes Ansehen zu verschaffen « ${ }^{74}$. Die Biblio-

71 Fouquet, Euvres, Bd. VI, S. 138.

72 Weiter heißt es: »Et apres s'estre arrestés cinq ou six heures dans la bibliotheque, et s'estre un peu promenés dans le jardin, ils nous ont affirmé, faisant en latin des reflexions morales sur la chutte de Mr foucquet [sic], et frapant leur poitrine pour donner plus de foy a leurs dires, que Rex hispaniarum nihil tale habebat, que diront-ils en visitant la maison de Vaux «(Discours sommaire de ce qui s'est passé, et a été inventorié de plus remarquable a St. Mandé, BN ms. fr. 10958, fol.230v.). Nach der Verhaftung Fouquets erhielt auch der Jesuitenvater Deschampsneufs eine Sondergenehmigung für die Benutzung der Bibliothek von Saint-Mandé.

73 JoLLy, Bibliothèques françaises, S. 4.

74 NAudé, Advis, S.20f. 
thek zeigte, um mit Martin zu sprechen, »le désir de gloire de grands seigneurs mécènes \} ^ { 7 5 } \text { . Mit dem Ausbau und der Öffnung schuf sich Fouquet einen Hof } von gelehrten Gefolgsleuten und mehrte damit sein Bild von einem aufgeschlossenen Mann mit Beziehungen und Einfluss. Da Fouquet, wie auch bei dem Aufbau seiner Kunstsammlung, dem Vorbild und der Kulturpolitik Mazarins folgte, ist sie zugleich Ausdruck der Ambition des Finanzministers, sich als Mäzen mit der politischen Spitze Frankreichs messen zu können.

\subsection{Kuriositäten und Raritäten}

Die Kuriositätensammlung galt im Ancien Régime als ein Teil der Bibliothek. Sie war in denselben Räumen untergebracht und ergänzte den Buchbestand durch ihre vielseitigen Objekte, zu denen Münzen und Medaillen, Karten und Stiche, Gemälde und Skulpturen ebenso gehörten wie wissenschaftliche Instrumente und Kuriositäten aller $\mathrm{Art}^{76}$. Die Leidenschaft für das Sammeln von Objekten aller Art - ob Büsten oder Blumen, Antiquitäten oder Kuriositäten, Medaillen oder Roquaillen - wuchs im Laufe des 17. Jahrhunderts. Im Jahre 1648 fanden sich in Paris bereits mehr als fünfzig cabinets curieux »à une époque où Rome n'en possède que huit [...] et Londres cinq [...]. La curiosité pénètre dans toutes les classes et dans tous les mondes «77. Bonnaffé zählt auch Nicolas Fouquet zu den amateurs de l'ancienne France, der die verschiedenen Formen der curiosité, der Liebhaberei seltener Gegenstände, in seinen zahlreichen Sammlungen vereinte und selbst von den Inventaristen nach 1661 als omnium curiositatum explorator bezeichnet wird ${ }^{78}$. In SaintMandé fanden sich fast sämtliche »grippez à la mode «, die in einer anonymen Quelle der Zeit aufgelistet werden: die »grippé des médailles, de la roquaille, de tableaux, des miroirs, des verres, des pierreries et des fleurs « ${ }^{79}$.

Zwei Stücke machten die Kuriositätensammlung des Finanzministers über die Grenzen von Paris hinaus berühmt: die ersten ägyptischen Mumien nebst ihren Sarkophagen, die ein französischer Händler 1632 nach Frankreich gebracht hatte. Fouquet kaufte sie im Jahre 1659, ließ sie nach Saint-Mandé bringen und stellte sie in der Galerie auf. Die »deux grands corps égyptiens tous entiers embaumés et en momie« verschwanden »dans un appentis fermé à clef, tout rempli de statues, de tables de marbre et de bronze ${ }^{80}$. Laut Inventar vom 26. Februar 1666 soll es sich bei den Mumien um »un roy et une reyne

75 Martin, Livre, Bd. I, S. 480.

${ }^{76}$ Erst nach der Französischen Revolution wurde zwischen Büchern und Objekten getrennt (JoLLY, Bibliothèques françaises, S. 6).

77 Bonnaffé, Foucquet, S. $8 \mathrm{f}$.

78 Discours sommaire de ce qui s'est passé, et a été inventorié de plus remarquable a St. Mandé, BN ms. fr. 10958, fol. 231r.

79 BN ms. fr. 12491, fol. 268.

${ }^{80}$ Discours sommaire de ce qui s'est passé, et a été inventorié de plus remarquable a St. Mandé, BN ms. fr. 10958, fol.231r., oder auch BN ms. fr. 17398, fol.115v. 
d'Egypte « gehandelt haben, die von La Fontaine als Kiopès und Céphrim identifiziert wurden:

En ce superbe appartement,

Où l'on a fait d'étrange terre,

Depuis peu, venir à grand'erre

(Non sans travail et quelques frais)

Des rois Céphrim et Kiopès

Le cercueil, la tombe ou la bière;

Pour les rois, ils sont en poussière,

C'est là que j'en voulois venir.

Il me fallut entretenir

Avec ces monuments antiques,

Pendant qu'aux affaires publiques

Vous donniez tout votre $\operatorname{loisir}^{81}$.

La Fontaine erwähnt nicht nur den Wert der Mumien. Aus seinen Versen geht zugleich hervor, dass die raren Stücke einem ausgesuchten Publikum zugänglich waren. Das Zitat lässt sogar noch eine weitere Schlussfolgerung zu: La Fontaine zufolge hat Fouquet die wertvollen Mumien ausschließlich für die Öffentlichkeit gekauft, denn die Staatsaffären ließen ihm keine Zeit für derartige Zerstreuungen.

Darüber hinaus fanden sich in Saint-Mandé zahlreiche antike Statuen und Büsten, die in der »Prisée des bustes étant à Saint-Mandé« verzeichnet sind ${ }^{82}$. Auch Muschelkunstwerk zeugte von der curiosité des Hausherrn. In den Aufzeichnungen zu Berninis Aufenthalt in Paris im Jahre 1665 berichtet Fréart de Chantelou von einer »grande coquille pleine de dieux marins, faits de corail« aus dem Besitz Fouquets ${ }^{83}$.

Das Sammeln von Medaillen gehörte ebenfalls zu den großen »grippez à la mode«. Eine anonyme zeitgenössische Quelle, »[qui] met en scène tous les grippez du jour « ${ }^{84}$, beschreibt den Reiz einer Münzsammlung wie folgt:

Dedans mes médailles de cuivre

Je lys les belles actions.

Des héros, et je fais revivre

Les Césars et les Scipions.

Pour les morts seuls j'ai ceste envie

De priser les antiquités;

Mais pour celles qui sont en vie,

J'adore les jeunes beautés ${ }^{85}$.

81 La Fontaine, Épître, in: La Fontaine, CEuvres complètes II, Bd. II, S. 502f. Die Mumien wurden später als Hor-Kheb und Ank-mer identifiziert, zwei unbekannte Personen der 26. Dynastie. Sie befinden sich heute im Louvre (D 5 \& D 7), siehe auch: Bonnaffé, Foucquet, S.19f.

82 AN O $^{1}$ 1964, abgedruckt in: BonNAFFé, Foucquet, S. 61-64. Auf die Kunstsammlung des Finanzministers wird weiter unten sowie in Kapitel 3.2 eingegangen.

83 Fréart de Chantelou, Journal du voyage, S.205f. Bernini bewertet die als Geschenk für den König gedachte Kuriosität als wertlos - »sans maîtrise ni excellence d'art, comme de petits bamboches «.

84 Bonnaffé, Foucquet, S.7.

85 BN ms. fr. 12491, fol. 268. 
Die Medaillen und ihre Ikonographie waren die Repräsentanten par excellence des invisible, das den ideellen Kern der Sammlungen ausmachte. Auf ihnen waren vergangene Zeiten und Helden dargestellt, die für die zeitgenössische Welt verloren waren und einzig durch ihre Aufbewahrung bei dem Sammler zurückgebracht werden konnten. Doch die Medaillensammlung gewann ihr Ansehen nicht allein durch den Bezug auf die Antike. Zugleich galten die Münzen als Sinnbild der Zusammenführung von Kunst und Wissenschaft, da sich durch die Vereinigung von Devisen und Abbildungen die Arbeit der Gelehrten mit jener der Künstler zu einem präzisen Kunstwerk verband.

Wie bei der Bibliothek konnte Nicolas Fouquet auch im Fall seiner Medaillensammlung an das Erbe seines Vaters anknüpfen, welcher eine »collection de médailles importantes ${ }^{86}$ besessen hatte. Peiresc, »[qui] ne manquait jamais, en voyage, de visiter les cabinets célèbres et d'en noter les particularités les plus intéressantes«, berichtet um 1616 von einem Besuch bei François Fouquet und »décrit longuement ses médailles romaines en or et en argent $\ll^{87}$.

Nicolas vergrößerte die Sammlung seines Vaters nur um wenige Stücke. In Saint-Mandé war die Kollektion »dans l'une des Chambres de la Bibliotheque « untergebracht ${ }^{88}$. Die »Estimation « der Münzen aus dem Jahre $1666^{89}$ zählt 57 Goldmedaillen, darunter 31 aus der römischen Kaiserzeit vor dem Jahr 476 n. Chr. und 26 Medaillen aus der Zeit danach, »y compris une pièce carrée aussi d'or de la Chine «90, sowie eine nicht näher bestimmte Menge an Silbermedaillen, Medaillen aus einem Silbergemisch (médailles de billon), Bronzemedaillen und $30 »$ médailles de cuivre un peu plus curieuses «. Die Medaillensammlung des Finanzministers umfasste über die in der »Estimation« aufgezählten Münzen hinaus zahlreiche Prägungen seiner Familie. Mit diesen konnte der kostbaren Sammlung eine persönliche Note gegeben und seinen Taten ein ewiges Denkmal gesetzt werden. Der Besitzer konnte nicht mehr nur in direkten Zusammenhang mit den heldenhaften Taten aus der Antike gebracht werden, sein reelles Leben war nun sogar Gegenstand der Münzen. Die eigene Münzprägung war ein weiteres Mittel, um sich gesellschaftlich abzuheben. Bereits sein Vater François Fouquet hatte zum eigenen Gedenken zwei Münzen schlagen lassen, auf denen sowohl das Eichhorn als auch die

86 SChNAPPER, Curieux, S. 215.

87 Manuskript des Museums Meermanno-Westreemanum in Den Haag, zitiert nach: BonNAFFÉ, Foucquet, S.12, Anm.2. Die Jahreszahl 1616 ist aufgrund von Schäden am Manuskript nicht eindeutig lesbar. Bonnaffé weist darauf hin, dass es sich auch um das Jahr 1610 handeln könnte.

${ }^{88}$ Discours sommaire de ce qui s'est passé, et a été inventorié de plus remarquable a St. Mandé, BN ms. fr. 10958, fol. 230v.

${ }^{89}$ AN O$^{1} 1964$, abgedruckt in: BonnafFé, Foucquet, S. 65-67. Die Medaillensammlung wurde 1666 auf den geringen Wert von 1040 Livres geschätzt.

90 AN O$^{1}$ 1964, abgedruckt in: BonNAFFé, Foucquet, S. 67. 
Devise »Quo non ascendet? « zu sehen sind ${ }^{91}$. Nicolas stellte gar einen eigenen graveur ein. Der aus Italien stammende Bertinetti fertigte etwa elf Münzen für Nicolas Fouquet an, die alle ein besonderes Ereignis zum Anlass haben und die familiären Feste in eine Verbindung mit den Staatsereignissen bringen. Die Münzen haben Fouquets Ernennung zum surintendant des Finances und seine Hochzeit mit Marie-Madeleine de Castille ebenso zum Inhalt wie den Friedensvertrag zwischen Spanien und Frankreich oder die Vermählung Ludwigs XIV. Auch sie zeigen das Wappentier und Fouquets Devise und darüber hinaus jene seiner zweiten Gattin ${ }^{92}$. Die Stellung des Münzprägers in der Entourage Fouquets wird an der Tatsache deutlich, dass auch er nach dem Sturz des Finanzministers in Haft genommen wurde. Damit hatte man seine propagandistische Arbeit für Fouquet jedoch nicht beenden können: »Dans sa prison Bertinetti a modelé de souvenir un médaillon en bronze de $64 \mathrm{~mm}$, représentant Foucquet en son costume de surintendant, avec la calotte et la simarre «33.

$\mathrm{Zu}$ der Kuriositätensammlung des Ministers ist auch seine Sammlung exotischer Pflanzen zu rechnen, die in dem von Le Nôtre angelegten Garten untergebracht war ${ }^{94}$. Das Interesse Fouquets an Heilpflanzen, ihre Zubereitung und Wirkung, die sein Arzt in der apothicairerie testete und die Fouquet selbst nach seiner Verhaftung in einem eigenen Labor in der Festung Pignerol studierte, ging bei dem Finanzminister mit der Passion für florale Kuriositäten einher. Bereits seit langem scheint sich Fouquet für die Botanik interessiert zu haben, denn 1652 heißt es in einer Widmung:

Monseigneur, l'Agriculture a pour vous tant de charmes \& tant de delices, que vous ne dédaignez pas d'y occuper vos mains, \& d'y donner les plus precieux moments de vostre loisir. Vous cherchez dans l'innocence de la vie champestre des douceurs qu'on ne sçauroit rencontrer autre part ${ }^{95}$.

Doch auch die Leidenschaft für Blumen und Pflanzen ist keine individuelle Vorliebe, sondern unter den Sammlern des Grand Siècle weit verbreitet und

91 Die Münzen befinden sich heute im Cabinet des Monnaies et Médailles der Bibliothèque nationale. Eine genaue Beschreibung sowie Abbildungen finden sich bei EsNaUlT, Armes, S. 102-120.

92 Die zweite von Esnault beschriebene Münze aus der Série 5 (Nicolas Foucquet) zeigt auf der Rückseite den »tour donjonnée de trois tourelles«. Die zehnte Medaille trägt auf ihrer Rückseite die Devise der Familie Castille »Surgit radicibus altis « (Il s'élève à partir de racines profondes). Ob die Medaillen tatsächlich alle aus der Hand Bertinettis stammen, ist nicht gesichert (ibid., S. 102).

93 GRÉsy, Graveur, S. 101.

94 SCHNAPPER, Géant, S. 40: »Le type idéal de la collection sous sa première forme est le jardin botanique, d'allure scientifique et médicale [...]; à l'autre catégorie appartient le passionné de tulipes [...], ou le spécialiste des anémones [...]. Mais les corrélations entre les deux types sont évidentes: même les jardins botaniques sont contaminés par la mode de certaines fleurs $\ll$.

95 Mollet, Claude, Theatre des plans et iardinages contenant des secrets et des inventions incognuës, hg. von Charles DE SERCY, Paris 1652, S. III. Es handelt sich hierbei um die erste Buchwidmung an Nicolas Fouquet. 
diente als weiteres Mittel der Distinktion in einer Gesellschaft, in der immer breitere Schichten sich den Luxus prunkvoller Häuser und großer Sammlungen leisten konnten ${ }^{96}$. Nach Schnapper zeichnete sich der collectionneur de fleurs im 17. Jahrhundert vor dem einfachen Blumenliebhaber durch »le désir passionné d'avoir le plus grand nombre possible de variétés de la même plante « aus ${ }^{97}$. Die Traktate der Zeit nennen bis zu 451 Varietäten von Tulpen und 77 von Anemonen ${ }^{98}$. Auch Fouquet trat in diesen botanischen Wettstreit ein. Dank der Lieferungen aus Italien konnte sich der Finanzminister im Jahr 1656 der größten Varietätensammlung von Anemonen erfreuen, was seinem Bruder Louis die Bemerkung entlockte: »Vous serez peut-être mieux pourvu en anémones qu'homme de France « ${ }^{99}$.

Louis war es auch, der für einen Austausch mit den großen italienischen Gartenfreunden sorgte. Am 24. April 1656 schrieb er aus Rom:

Je vous envoye une boite de greffes d'orangers et de citronniers des plus rares d'Italie; je les ay recouvrés pour rien dans les deux plus célèbres jardins de Rome. Ils sont acommodés avec du miel et de la mousse et se doivent conserver. Il faut les enter sur citronniers ou orangers indifféremment. J'establiray avant mon départ des commerces avec les plus illustres amateurs de Rome, à qui vous pourrez envoyer des raretés de France et recevoir de celles d'Italie. Mgr Senci, qui a le plus beau jardin d'Europe en agrumes, est celuy qui en a donné la meilleure partie, et un père Ferdinand le reste ${ }^{100}$.

Wie sonst nur beim Kauf von Büchern achtete Fouquet bei den Blumen und Pflanzen auf Exklusivität und Originalität und betrieb einen hohen Aufwand, um an seine botanischen Kuriositäten zu gelangen. So wundert es nicht, dass Regnard Fouquets Gärtner Besseman als den wichtigsten Mann in SaintMandé bezeichnet:

Le jardinier de Saint-Mandé qui est vêtu, logé et meublé comme un honnête homme, et que l'on appelle Le Fleuriste est celuy à ce qu'on m'a dit, celuy de tous les domestiques dudit lieu duquel le sieur Fouquet faisait le plus destat, et auquel il prenait le plus de confiance, nonobstant qu'il fut allemand luthérien, qui a sous luy trois ou quatre Lutheriens ${ }^{101}$.

$96{ }$ C'est dans les [...] années 1650-1670 que la manie des fleurs et des fruits semble à son comble « (SchnapPer, Géant, S. 355). In dieser Zeit steigt auch die Anzahl der Veröffentlichungen zur Pflanzenkunde rapide an. Schnapper vermerkt außerdem: »Le goût des fleurs est associé à des curiosités plus attendues, comme celle des médailles ou celle des estampes: les mêmes personnages réunissent souvent les trois grandes catégories de la collection au XVIIe siècle, singularités de la nature, témoignages de l'Antiquité, œuvres d'art « (ibid., S.357).

97 SCHNAPPER, Fleurs, S. 354.

98 LA Quintinie, Jean de, Instructions pour les jardins fruitiers et potagers, Paris 1700. Die Erstausgabe erschien 1690, ihr fehlte noch die der Ausgabe von 1700 beigefügte »Nouvelle instruction pour la culture des fleurs contenant la maniére de les cultiver [...] avec un catalogue des fleurs les plus belles \& les plus rares«.

${ }^{99}$ Louis Fouquet an Nicolas Fouquet am 24. April 1656 aus Rom; in: FouQuet, Lettres, S.306.

100 Ibid.

101 Discours sommaire de ce qui s'est passé, et a été inventorié de plus remarquable a St. Mandé, BN ms. fr. 10958, fol. 230r. 
Besonders die Zitrusbäume erforderten nicht allein einen kompetenten Gärtner, sondern auch einen eigenen Bau, der sie im Winter vor der Kälte schützte ${ }^{102}$. Den exotischen Schatz, rund 200 Bäume, ließ Ludwig XIV. nach dem Sturz des Finanzministers nach Versailles bringen ${ }^{103}$.

Daneben war der Garten von Saint-Mandé ein Ort für medizinische Studien. Der Kräutergarten bot dem Mediziner Pecquet Nährboden für seine Experimente, die er in der apothicairerie durchführte. Jean Pecquet (1622-1674), »[qui] travailla sur le rôle des vaisseaux chylifères pendant la digestion et sur la composition des eaux minérales « ${ }^{104}$ und der durch seine Beschreibung des canal thoracique berühmt geworden war ${ }^{105}$, stand mit großer Wahrscheinlichkeit schon vor 1648 in Fouquets Diensten ${ }^{106}$. Als domestique de la maison verfügte er in Saint-Mandé über ein eigenes Appartement und übernahm Aufgaben eines maître d'hôtel ${ }^{107}$. Ob die Förderung Pecquets durch Fouquet als mécénat scientifique bezeichnet werden kann, ist $\mathrm{zu}$ bezweifeln: »On ne touche vraiment au mécénat que lorsqu'un homme de science est >domestique< d'un grand personnage. Encore faut-il demeurer prudent. Le protecteur n'a jamais choisi son protégé sur la seule considération de sa qualité de savant, mais pour son aptitude à remplir certaines fonctions « ${ }^{108}$. Dank Kräutergarten und Bibliothek mit ihren rund 273 librj Medicj ${ }^{109}$ konnte Pecquet in Saint-

102 Schnapper, Géant, S. 51. Als einziger Gebäudeteil ist die Orangerie auf dem Stockholmer Plan als solche ausgezeichnet. Sie befindet sich in dem südlichen Flügel der Anlage.

$103 \mathrm{AN} \mathrm{O}^{1} 1964, \mathrm{n}^{\circ} 16$ (2): Prisée des orangers estant à St. Mandé du 26 février 1666.

104 Michel, Vatel, S.38. Guy Patin, der allgemein schlecht auf Pecquet zu sprechen war, schildert die Arbeit des Mediziners in Saint-Mandé in einem Brief an Sorbière vom 6. November 1657 folgendermaßen: »M. Pecquet s'amuse à distiller des eaux minérales de diverses fontaines [...] il ne fait autre chose et [...] n'écrit rien du tout « (PATIN, Lettres, Bd. II, S.353).

105 Pecquet, Experimenta.

106 In einem Brief vom 3. August 1648 an Mersenne bittet er diesen »d'envoyer vos lettres au R.P. des Champsneufs ou chez Monsieur fouquet [sic] Maistre des requestes Rue des Mauvais garcons« (BN ms. fr. n. a. 6205, fol. 157).

107 Pecquet hat wiederholt für Fouquet Rechnungen bezahlt, die Ausgaben für SaintMandé beglichen. Die Prozessakten Fouquets geben über Rückerstattungsanträge Pecquets Auskunft, die er aufgrund von Lohnzahlungen an Angestellte Fouquets in SaintMandé forderte.

108 Mesnard, Mécénat scientifique, S.109. Auf Seite 107 heißt es zudem: »On ne peut parler de mécénat qu'au cas où une aide matérielle, principalement pécuniaire, est accordée aux hommes de science: assurance de vivre et du couvert, octroi des subventions ou des pensions, prise en charge des frais de la recherche. [...] Qu'un grand seigneur entretienne dans sa maison un médecin: il n'en pratique pas pour autant le mécénat scientifique «.

109 BN ms. fr. 9438: Inventaire, Prisée \& Estimation des livres trouvés A St. Mandé, fol. 40-46. Der Bestand an medizinischer Fachliteratur fand im Jahre 1657 eine große Ergänzung, als Fouquet einen Teil der Bibliothek des verstorbenen Mediziners Moreau aufkaufte: »Les quatre libraires qui avoient acheté la bibliothèque de feu M. Moreau avoient arrangé les livres dans une belle boutique de la foire pour les vendre lundi prochain; mais ils en sont soulagés de moitié. M. Foucquet, procureur général, a acheté les livres de mé- 
Mandé zwar seinen Forschungen nachgehen. In erster Linie aber war er Hausarzt. Der surintendant litt unter häufigen Fieberattacken und einer allgemein schlechten gesundheitlichen Verfassung ${ }^{110}$. Zudem darf nicht vergessen werden, dass Nicolas Mutter eine bekannte Heilkundlerin war und Pecquets Studien vielleicht auf ihr Betreiben hin ermöglicht wurden, um auch ihr Wissen wachsen zu lassen. Eine Förderung der medizinischen Forschung im größeren Stil findet sich in Saint-Mandé also nicht, jedoch eine große curiosité für die Medizin.

\subsection{Kunstkäufe für Nicolas Fouquet}

Ein herrschaftliches Anwesen konnte erst in voller Pracht erstrahlen, wenn es mit entsprechenden Kunstwerken ausgestattet war, die Schönheit, Gelehrtheit und Poesie in Garten und Räume brachten. Die Deckenmalereien waren nur ein Teil der künstlerischen Ausgestaltung. Skulpturen, Gemälde und Tapisserien gaben dem Bau Exklusivität und waren durch ihre Ikonographie, die meist biblische oder mythologische Themen aufgriff, ein Zeichen der Bildung ihres Besitzers. Nicht zuletzt diente die Kunstsammlung auch dem Divertissement der Schlossbewohner, denen der Kunstgenuss als mentales Labsal diente. Mit Hilfe ferner Händler und eigens entsandter Agenten vermochten die Kunstliebhaber des Grand Siècle ihre Sammlungen durch Ankäufe in ganz Europa zu erweitern. Besonders der italienische Markt mit seinen reichen Schätzen war bei den französischen collectionneurs gefragt - sein Angebot jedoch sehr ausgesucht oder überteuert. Im Laufe des 17. Jahrhunderts gewannen daher die Märkte der Niederlande an Attraktivität.

Bis 1661 nahm die königliche Kunstsammlung einen sekundären Platz unter den Kollektionen Frankreichs ein. Wieder einmal waren es die Premierminister, die unter dem Deckmantel des staatlichen Mäzenatentums für sich selbst als Kunstsammler tätig wurden. Neben ihnen waren es die übrigen Minister, unter anderen Particelli d'Émery und Séguier, die dank ihrer guten Beziehungen und reichlicher finanzieller Mittel bedeutende Kunstsammlungen anlegten.

Auch das neue Anwesen Fouquets verlangte nach einer dem Rang des Hausherrn entsprechenden Ausstattung mit Kunstwerken. Mit dem Kauf und Ausbau von Saint-Mandé stellte der Finanzminister Künstler in seine Dienste, die für die malerische und skulpturale Ausschmückung des Schlosses sorg-

decine, qu'il a fait enlever aussitôt et emporter chez lui, moyennant 10000 livres d'argent comptant« (PATIN, Lettres, Bd. II, S.276, in einem Brief an Charles Spon vom 16. Februar 1657).

110 Patin, Correspondance, S. 255 in einem Brief an Charles Spon vom 5. Juli 1658: »Nous avons ici un de nos magistrats bien malade, qui est $M$. Fouquet, procureur général et surintendant des finances. Oh! Belle chape-chute, si cette asme montonnière et loyolithique se laissait mourir! Mais cela n'arrivera point car il est encore jeune, il a les dents et les ongles fort bons «. 
ten. Charles Le Brun wurde mit der Deckenbemalung beauftragt ${ }^{111}$. Vielleicht aus Mangel an antiken Originalen, vielleicht aber auch aus Gefallen an den zeitgenössischen Skulpturen beschäftigte Fouquet französische Bildhauer; 1655 engagierte er Michel Anguier, der für Saint-Mandé vierzehn Götterfiguren in Brontia (pierre de Tonnerre) für die Galerie sowie eine Flora aus Marmor und eine sitzende Geometrie für den Garten ausführte ${ }^{112}$. Guillet de Saint-George schreibt Anguier zudem eine Herkulesfigur sowie eine »Charité« zu, die Mme Fouquet darstellt, im rechten Arm ein schlafendes Kind haltend, ein weiteres zu ihren Füßen und zwei Kinder spielend in ihrer Nähe »pour [...] marquer la tendresse et l'union qui régnoient dans cette famille «113. Die »Prisée des bustes étant à Saint-Mandé« von 1666 verzeichnet neben Skulpturen von Michel Anguier einen weiteren zeitgenössischen Skulpteur und seine Arbeit für Saint-Mandé: Jacques Sarrazin und eine nicht vollendete Venus ${ }^{114}$.

Neben den Aufträgen, die vor Ort ausgeführt wurden, ist der Kunsterwerb durch Fouquet ab dem Jahr 1655 durch einen Briefwechsel mit seinem Bruder Louis belegt. Im Sommer nach dem Kauf von Saint-Mandé sandte Nicolas Fouquet den Abbé nach Rom ${ }^{115}$. Dieser sollte dort nicht nur diplomatisch tätig werden und M. de Lionne, den Botschafter des Königs, ausspionieren, sondern zudem »acheter, pour l'ornement des maisons de [...] Saint-Mandé et Vaux, tout ce qu'il pourrait trouver de plus beau dans la ville éternelle en tableaux, statues et meubles précieux «116. Die Wahl der Themen und Künstler der Werke überließ Nicolas seinem Bruder. Vor Ort konnte dieser sich, vor allem was die Finanzierung und Ausfuhr der gekauften Objekte anging, auf Agenten des Finanzministers stützen ${ }^{117}$. Bei der Sichtung der Kunstgegenstände sollte er Kontakte zu ortsansässigen Künstlern und Sammlern knüpfen, die als Subagenten dienten.

$111 \mathrm{Zu}$ den Arbeiten Charles Le Bruns in Vaux-le-Vicomte: siehe Kapitel 3.1.

112 Prisée des bustes étant à Saint-Mandé (1666), in: Bonnaffé, Foucquet, S. 63.

113 Guillet de Saint-George, Mémoires, Bd. I, S. 440.

114 Prisée des bustes étant à Saint-Mandé (1666), in: Bonnaffé, Foucquet, S. 63.

115 Die die Kunstkäufe betreffende Korrespondenz wurde 1862 von Lépinois in den Archives de l'art français veröffentlicht (FouQueT, Lettres, S.267-309). Erhalten sind die Briefe Louis Fouquets aus Rom, jedoch nicht die Schreiben Nicolas Fouquets an seinen Bruder. Die Briefe zu den Kunstkäufen befinden sich in Privatbesitz und sind Teil einer umfangreichen Briefsammlung. Lépinois nennt 1862 M. de Cossé-Brissac als Eigentümer. 116 M. A. de Montaiglon, in: ibid., S.268.

117 Aus den Briefen Louis' wissen wir, dass M. Bernard als Financier in Rom für Fouquet tätig war: »J'ay tiré, cet ordinaire, une lettre de change de 2130 livres, le change compris, sur M. Bernard « (Brief Louis Fouquets an Nicolas vom 24. April 1656, in: ibid., S. 305). Die Ausfuhr erfolgte über verschiedene Mittelsmänner. So schreibt Louis am 3. April 1656 in Bezug auf die zweite Sendung aus Rom an seinen Bruder »Je vous envoie 14 tableaux par la voie de M. de Lionne« (ibid., S.303). Die folgende Lieferung soll über einen anderen Mittler versendet werden: »J'espère de vous envoyer par M. d'Argenson une table magnifique de pièces rapportées« (Brief vom 17. April 1656, in: ibid., S.305). 
Mit Hilfe von Nicolas Poussin und dank der Vermittlung von Paul Fréart de Chantelou wählte der Bruder zahlreiche Objekte, vor allem Statuen, aber auch Gemälde und Kunsthandwerk aus, die er in den folgenden Monaten nach Frankreich bringen ließ. Bereits kurz nach seiner Ankunft in Rom entschied sich Louis, seine Aufmerksamkeit vor allem auf Skulpturen zu konzentrieren: »[Les statues] parent incontestablement davantage de grands appartements que de misérables tableaux, dont peu de gens sont capables degouster les beautés «118.

\subsubsection{Die Skulpturensammlung}

Der italienische Markt bot ein reichhaltiges Angebot an Skulpturen aller Art und Preisklassen. Louis entschied, entweder unversehrte antike Statuen oder moderne Repliken der alten Meister zu erwerben, »en sorte que toutes les testes, par exemple, que j'envoyeray se pourront toutes reconnoistre par les habiles, ou sur les médailles ou par les idées des anticques qu'ils auront veus dans Rome «119. Von seinem Bruder erhielt er darüber hinaus die Anweisung, Basreliefs sowie eine große Quantität an Objekten zu kaufen, die auch unter freiem Himmel aufgestellt werden konnten ${ }^{120}$.

Der Kauf antiker Skulpturen hatte für Louis Fouquet Vorrang, wenngleich deren Markt undurchsichtig blieb und die Objekte nur selten ohne Schäden waren. Der Vorsatz, nur unversehrte oder aber gut restaurierte Skulpturen zu kaufen, ließ sich kaum umsetzen. Louis wandte sich daher an Restaurateure, die die gekauften Objekte vor dem Transport in einen guten Zustand bringen sollten $^{121}$. Die Künstler brachten die »Restauration « der Skulpturen, die aus der Hinzufügung von Fingern, Füßen oder Nasen, aber auch aus der kompletten Wiederherstellung eines Torsos bestehen konnte, zu einer solchen Fertigkeit, und die künstlerischen Eigenarten der Bildhauer beeinflussten das Bild der Zeitgenossen von den antiken Statuen mit der Zeit derart, dass es schwierig wurde, Originale von Repliken zu unterscheiden. Zumindest in ihrer Jugend hatten alle in Rom ansässigen Skulpteure sich in dieser Kunst geschult. Diese hohe Kunstfertigkeit mag der Grund dafür gewesen sein, dass Louis die von ihm gekauften Statuen vor Ort restaurieren ließ.

Der Abbé gibt in seinen Briefen nur zwei direkte, leider aber sehr unpräzise Hinweise auf den Erwerb von Statuen. Am 10. April 1656 berichtet er von dem Kauf einer »bellissime statue antique«, vierzehn Tage darauf von einer »statue restaurée«, die er für seinen Bruder erworben habe ${ }^{122}$. Die Beschreibung seiner ersten Sendung nach Paris - sowie die Vielzahl der in den Inventaren angeführten antiken Skulpturen - lässt jedoch vermuten, dass Louis

118 Brief Louis Fouquets vom 2. August 1655 und vom 24. April 1656, in: ibid., S. 288f.

119 Brief Louis Fouquets vom 2. August 1655, in: ibid., S. 289.

120 Brief Louis Fouquets vom 23. August 1655, in: ibid., S. 292.

121 Brief Louis Fouquets vom 10. April 1656, in: ibid., S. 303.

122 Ibid., S. 303, 305. 
weit mehr Erfolg bei seinen Einkäufen hatte, als dies seine wenigen Bemerkungen vermuten lassen.

Zunächst verzögerte die Schwierigkeit, eine Ausfuhrerlaubnis für die erworbenen Skulpturen zu erhalten, deren Versendung. Beim Export antiker Skulpturen waren in Rom bereits seit dem 15. Jahrhundert erhebliche Hindernisse zu überwinden: »La permission de transporter les anticques a esté quelquefois impossible à obtenir et jamais n'est aysée «123. Als Louis Ende Dezember 1655 endlich die ersehnte Zustimmung des Papstes erhalten hatte, waren nurmehr die Transportprobleme zu lösen. Insgesamt sind drei Sendungen nach Paris belegt. In einem Brief vom 11. Januar 1656 berichtet der Abbé von der ersten Sendung mit Statuen, Büsten, Fußgestellen, Möbeln, Gemälden und Teppichen. Am 3. April spricht er von einer zweiten und am 17. April kündigt er eine dritte Sendung an. Die bis Januar 1656 erworbenen Statuen, Büsten und Fußgestelle von großem Gewicht und hoher Zerbrechlichkeit wurden auf dem Seeweg bis nach Saint-Malo verschifft. Die Möbel, Gemälde und Teppiche konnten ab Marseille den Landweg nehmen ${ }^{124}$.

Louis Fouquet, »[qui] se sentait moins habile en peinture qu'en diplomatie ${ }^{125}$, hätte sich in dem überteuerten und undurchsichtigen römischen Kunstmarkt nicht ohne die Hilfe von Nicolas Poussin zurechtfinden können, der ihn nicht nur künstlerisch beriet, sondern auch die Käufe vermittelte und zum Teil persönlich tätigte: »Je ne puis estre plein de reconnoissance de tous les soins que la famille toute entière de M. Poussin prend des choses que je souhaite «126. Für den alten Meister war die Bindung an das Haus Fouquet nicht unwichtig. Seit vielen Jahren versuchte er, sich den Titel des peintre $d u$ $R o i$ von der französischen Krone bestätigen zu lassen und sein Gehalt für die im Jahre 1643 geleistete Arbeit am Hof ausbezahlt zu bekommen ${ }^{127}$. Louis bemühte sich, sich für den zuvorkommenden Künstler einzusetzen und erreichte bei seinem Bruder über die genannte Anerkennung und Zahlung hinaus auch ein persönliches Schreiben, das der Finanzminister nach Rom sandte. Dies verstärkte die Bindung zwischen Louis und Nicolas Poussin, der sich anbot, für Fouquet Auftragsarbeiten auszuführen. Ein Rätsel bleibt die Andeutung

${ }^{123}$ Louis in einem Brief an seinen Bruder vom 16. August 1655, in: ibid., S.290. Einem Brief vom 29. Februar 1656 zufolge benötigte man für den Erhalt einer Ausfuhrerlaubnis zunächst die Erlaubnis des Papstes, anschließend die Zustimmung des commissaire des visites pour les anticques, dann jene des cardinal camerlingue, der Chambre apostolique, die Genehmigung durch den Zoll, die Übereinkunft mit der Post und dem Kapitän des Schiffes, der die Ware nach Frankreich bringen sollte. Louis bemerkte hierzu: »Il est absolument impossible que l'on achète ici quelque chose de la nature de celles qui ne sortent qu'avec licence« (Brief Louis Fouquets an Nicolas Fouquet vom 29. Februar 1656, in: ibid., S. 298f.).

124 11. Januar 1656, in: ibid., S. 298.

125 Ibid., S. 268.

126 Brief Louis Fouquets vom 2. August 1655, in: ibid., S.289.

127 »Je n'ai rien touché de l'année quarante-trois, laquelle j'emploiay toutte pour les desseins de la Gallerie « (Poussin in einem Brief an Fréart de Chantelou vom 20. Dezember 1655, zitiert nach: ibid., S.296). 
Louis’ vom 17. April 1656, er habe mit Poussin ein Projekt in Planung, »qui vous donneront par M. Poussin des avantages (si vous ne les voulez pas mespriser) que les roys auroient grande peine à tirer de luy, et qu'après luy peut estre personne au monde ne recouvrera jamais dans les siècles advenir «128. Um die einzige Skulpturengruppe aus Poussins Hand ${ }^{129}$ kann es sich nicht gehandelt haben, da diese zu diesem Zeitpunkt bereits in Arbeit war.

Um die skulpturalen Arbeiten in den Schlössern und Gärten Fouquets voranzutreiben, schlug Louis im August 1655 die Anwerbung zweier römischer Bildhauer von Rang und Namen vor:

Je suis persuadé que le signor Orphée, qui est le meilleur sculpteur de Rome après le cavalier Bernin, tient ici à peu de chose, et qu'il auroit mesme démangeaison de venir travailler en France, s'il y estoit aydé ou soustenu. Vous pourriez [...] l'attirer sous le nom du roy pour travailler au Louvre, et ensuite on l'appliqueroit aysément à des ouvrages particuliers [...]. Il n'est rien de plus habile que ce sculpteur pour la science de son art et pour l'invention. L'exécution [...] n'est pas plus belle, à ce que disent les Romains, que celle du signor Bernardo, qui aussi bien qu'Orphée travaille pour vous ${ }^{130}$.

Über Käufe antiker Skulpturen in Frankreich durch Fouquet ist nur wenig überliefert. Im eigenen Land war der Markt hierfür nicht gegeben. Aus Louis' Briefen wissen wir, dass Paul Fréart de Chantelou dem Finanzminister den Verkauf von zwölf antiken Köpfen angeboten hatte, denn der Bruder bittet aus Rom »que j'en eusse les noms, pour esviter d'en prendre de semblables ou d'en faire tirer sur les originaux « ${ }^{131}$. Ob Fouquet diese tatsächlich erwarb, bleibt unklar.

\subsubsection{Die Gemäldesammlung}

Aus gutem Grund hatte sich Louis dafür entschieden, in Rom vor allem nach Skulpturen Ausschau zu halten: »Ces chertés de tableaux me réduisent dans les bustes et dans les statues. [...] Je crois qu'il faut que le fort de vostre dépense aille en statues $\ll^{132}$.

Mitte des 17. Jahrhunderts war der italienische Kunstmarkt vollständig ausgereizt. Nicht nur in Frankreich galten die italienischen Werke vor allem des Cinquecento als die vollkommensten. Die Preise der toten Meister bewegten sich auf hohem Niveau, besonders begehrt waren die Werke Raffaels, die so gut wie unbezahlbar waren. Für die französischen collectionneurs attraktiv

\section{Ibid., S. 304.}

129 Nicht bedacht werden an dieser Stelle die unzähligen Wachsfiguren, die Poussin üblicherweise anfertigte, um den Körperbau und Faltenwurf der Figuren seiner Gemälde zu studieren.

130 Brief Louis Fouquets an seinen Bruder vom 23. August 1655, in: Fouquet, Lettres, S.293.

131 2. August 1655, in: ibid., S.289. Lépinois merkt an, dass es sich hierbei unter anderem um die von Poussin 1644 im Namen Fréart de Chantelous erworbenen acht antiken Köpfe aus der Sammlung Hippolyte Vitelleschis handeln könnte, unter denen sich ein Euripides und ein junger Augustus befanden (ibid.).

132 Brief Louis Fouquets an Nicolas Fouquet vom 2. August 1655, in: ibid., S. 288. 
war darüber hinaus der in Italien arbeitende französische Maler Nicolas Poussin.

Die Briefe Louis Fouquets aus Rom geben wiederholt Aufschluss über seine Schwierigkeiten, Gemälde zu akzeptablen Preisen zu finden, für die gekauften Kunstgegenstände eine Ausfuhrgenehmigung zu erhalten und überdies die Wünsche seines Bruders zu erfüllen. »Je ne crois pas que vous vouliez de ces originaux des premiers peintres du monde dont la cherté et la rareté est estonnante « ${ }^{133}$, schrieb er kurz nach seiner Ankunft in Italien im Juni 1655. Der römische Markt sei »plein de gens connoissants, on en a transporté ce qu'il y avoit de meilleur, et le peu qui y reste n'est plus dans le commerce «134. Die verbliebenen Gemälde seien übermäßig teuer und ein Kauf daher wenig attraktiv. Doch anscheinend wollte der Finanzminister nicht ganz auf die erwünschten dekorativen tableaux verzichten, denn wenige Wochen später schrieb ihm sein Bruder aus Rom: »Comme j'ai prévu que ce seroit un de vos souhaits, je ne cherche que de grands tableaux, propres à grands appartements, et j'y ajoute de moy encore une autre règle que, tant que faire se pourra, ce ne soient que choses prophanes, ou histoires de l'Ancien Testament « ${ }^{135}$.

In den ersten vier Monaten begutachtete der Abbé mehr als zweitausend Bilder »et de ces deux mille j'aurois honte d'en avoir emporté plus de vingt, quand presque j'aurois eu le surplus pour rien ${ }^{136}$. Kein einziges hatte er zu kaufen gewagt, da entweder ihr Preis oder schlechter Zustand (er spricht an dieser Stelle nicht, wie der Agent Mazarins in Rom, von der Angst, übers Ohr gehauen zu werden) ihn abgeschreckt hatten. Allein Poussins Bilder, selbst auch überteuert und meist unverkäuflich, schienen ihm von passender Qualität zu sein ${ }^{137}$. Im Sommer 1655 wurden auf dem italienischen Markt drei Werke des französischen Meisters zu je 2000 Livres zum Kauf angeboten, die die Preisvorstellungen der Fouquets weit übertrafen. Auch Jean Pointel, der seit 1654 in Rom war, um hier im Namen des Duc de Créquy Gemälde zu erwerben, hatte sich in eineinhalb Jahren zu keinem Kauf entschließen können. Im Dezember stand für Louis fest: »La difficulté est horrible pour des tableaux raisonnables, car M. Poussin ne se peut résoudre à m'en laisser prendre des médiocres «138. Obgleich er auf zwei Jahre im Voraus ausgelastet war, erklärte sich Poussin bereit, für den Finanzminister zu malen. Nicht ohne Begeisterung berichtet Louis seinem Bruder im August

133 Brief Louis Fouquets an Nicolas Fouquet vom 28. Juni 1655, in: ibid., S. 286.

134 Weiter heißt es: »De sorte qu'il faut des longueurs infinies pour attendre de favorables occasions de faire de raisonnables emplettes sans estre tyrannisé« (Brief Louis Fouquets an Nicolas Fouquet vom 2. August 1655, in: ibid., S.287).

135 Brief Louis Fouquets an Nicolas Fouquet vom 23. August 1655; in: ibid., S. 295.

136 Ibid., S. 294.

137 »On ne rencontre plus ici à achepter aucun passable tableau, hors ceux de M. Poussin, dont la cherté est estonnante (Brief Louis Fouquets an Nicolas Fouquet vom 2. August 1655, in: ibid., S.287).

138 Brief Louis Fouquets an Nicolas Fouquet vom 27. Dezember 1655, in: ibid., S. 297. 
von diesem Angebot: »On auroit plus de profit de luy faire faire quelques tableaux que d'en achepter de faits. Ses ouvrages, dans quelque temps après sa mort, seront mille fois plus rares et plus chers «139. Doch Nicolas konnte nicht warten - und war zudem nicht bereit, den (wenngleich geminderten) Preis für ein Gemälde von Poussin zu bezahlen. Er gab seinem Bruder Anweisung, eine möglichst große Menge mittelmäßiger Bilder zu erstehen, um seine Häuser zu füllen ${ }^{140}$.

Bereits im August 1655 hatte Louis Kontakt zu einer weiteren Malergröße aus Frankreich aufgenommen: Pierre Mignard. Auch bei ihm war sich der Abbé sicher, dass er zu Auftragsarbeiten für den Finanzminister bereit wäre. Wie bereits bei Poussin, so kam es aber auch in diesem Fall zu keiner Ausführung $^{141}$.

Ein halbes Jahr nach seiner Ankunft in Rom - Louis hatte bereits zahlreiche Objekte gekauft - gab er am 27. Dezember 1655 die Nachricht von der Zustimmung des Papstes für einen ersten Transport der Kunstgegenstände nach Paris weiter ${ }^{142}$. Im Januar 1656 kündigte er schließlich die erste Sendung mit Gemälden an, die bis zum 7. März nach Paris transportiert wurde ${ }^{143}$ :

Il y a une Décolation de saint Jean, un Massacre d'Abel, un Baptême de Notre-Seigneur, une Magdeleine, un Saint Jean l'évangéliste, un Saint François, un Saint Sébastien percé de flèches, un Saint Joseph, qui sont tous originaux, fort beaux et fort bien faits et de grands peintres tous morts. Vous y en avez encore trois, mais médiocres; un paysage de Gaspard, beau-frère de M. Poussin, et deux copies d'originaux de M. Poussin faites de bon main; elles représentent une Exposition de Moyse et une Vierge avec un Jésus ${ }^{144}$.

Einen Monat darauf folgten, mit der berühmten Anemonensendung, vierzehn weitere tableaux, die von Louis nicht näher benannt wurden ${ }^{145}$. Erstaunlich ist die Übereinstimmung der Anzahl der Gemälde dieser Sendung mit den 1671 in einem Untersuchungsantrag (requeste) des Königlichen Rates aufgeführten vierzehn Bildern, die in diesem Jahr zur Begleichung der Schulden Fouquets verkauft werden sollten. Ob es sich bei dieser Gemäldegruppe um die Sendung vom April 1656 handelt, bleibt fraglich, da sich unter den vierzehn tableaux neben Bildern von Paolo Veronese und der Familie Bassano auch

139 Louis Fouquet in einem Brief an Nicolas Fouquet vom 2. August 1656, in: ibid., S. 290.

140 »Je vous chercheray les meilleurs tableaux que je pourray entre ces médiocres que vous me demandez« (Louis Fouquet in einem Brief an Nicolas Fouquet vom 7. März 1656, in: ibid., S. 300).

$141 »$ M. Mignard, second peintre de Rome et le premier pour les portraits [...] entreprendroit pour vous quelque ouvrage « (Louis Fouquet in einem Brief an Nicolas Fouquet vom 2. August 1655, in: ibid., S. 293).

142 Ibid., S. 297.

143 »Les autres choses qui peuvent aller plus seurement, comme un lit de broderie non achevée [...], un tapis de Perse, un ballot de tableaux [...] iront par Marseille « (Louis Fouquet in einem Brief an Nicolas Fouquet vom 11. Januar 1656, in: ibid., S.298).

144 Brief Louis Fouquets an Nicolas Fouquet vom 7. März 1656, in: ibid., S.301. Zu der Zuordnung der Gemälde: siehe Anhang 2.

145 Brief Louis Fouquets an Nicolas Fouquet vom 3. April 1656, in: FouQuet, Lettres, S. 303. 
Gemälde der Niederländer Jan Brueghel d. Ä., Lambert Sustris und Anthonis Mor finden. Dass diese von Louis in Rom gekauft wurden, ist jedoch nicht in Gänze auszuschließen, da auch auf dem römischen Markt mit Werken der nordischen Schule gehandelt wurde und sowohl Lambert Sustris als auch Anthonis Mor einen Teil ihres Lebens in Italien verbrachten ${ }^{146}$. Eine dritte Sendung kündigt Louis in einem Brief vom 17. April an und fügt hinzu: »J'espère de conduire demain ou après-demain le marché de force beaux tableaux pour vous ${ }^{147}$. Es ist nicht bekannt, was aus diesem Einkauf geworden ist, denn der Briefverkehr zwischen den Brüdern brach am 24. April ab. Es bleibt allerdings zu vermuten, dass Louis bei seinem erneuten Gemäldeerwerb Erfolg hatte und weitere tableaux nach Paris schicken konnte. Im Frühsommer 1656 kehrte er nach Frankreich zurück. Mit der Rückkehr nahm seine kunstinspektorische Arbeit für Nicolas Fouquet ihr Ende.

Da die Anzahl der überlieferten Gemälde im Besitz Fouquets (rund 45) jene der Käufe von Louis in Rom (25) übersteigt, kann man annehmen, dass der Finanzminister auch den französischen Markt für Gemäldekäufe nutzte. Über mögliche Agenten Fouquets auf dem französischen Markt ist wenig bekannt. Thuillier vermerkt, dass der Financier und Kunstsammler Hesselin, der den Maler Sébastien Bourdon 1637 auf einer Reise durch Italien begleitete und dem man großen Sachverstand nachsagte, Fouquet bei seinen Kunstkäufen beriet ${ }^{148}$.

Dass der Finanzminister seine Sammlung - wie Mazarin - durch den Erwerb von Gemälden auf dem englischen Markt (der vor allem in den fünfziger Jahren durch zahlreiche Nachlassverkäufe die französischen Kollektionen bereicherte) vergrößerte, kann laut Cosnac indes ausgeschlossen werden: »Le surintendant Fouquet, bien que grand amateur de curiosités, ne se risqua pas à devenir le concurrent du cardinal Mazarin: il aurait eu trop à perdre à lui déplaire, s'il eut tenté de marcher sur ses brisées dans un terrain aussi restreint pour les beaux-arts que celui de l'Angleterre ${ }^{149}$.

Von der Möglichkeit, Gemälde bei zeitgenössischen Künstlern in Auftrag zu geben (zum Beispiel bei Poussin oder Mignard), profitierte Fouquet in der ersten Phase seiner Kulturpatronage nicht. Es existiert jedoch ein Bild Poussins aus den fünfziger Jahren des Jahrhunderts, das in das unmittelbare Um-

146 Michel, Mazarin, S. 250 weist zudem darauf hin, dass der römische Markt auch hinsichtlich der nordischen Schule ein reichhaltiges Angebot vorzuweisen hatte. In Bezug auf die Sammlung Mazarins vermerkt er: »La primauté de Rome est incontestable dans l'enrichissement des collections. Il est frappant de constater que les collections de la Ville éternelle sont susceptibles de répondre aux demandes du Cardinal, même en ce qui concerne la peinture nordique $\ll$.

147 FouQuet, Lettres, S. 305.

148 »Hesselin, qui paraît avoir conseillé Foucquet pour tout ce qui touchait aux cérémonies et aux œuvres d'art« (Thuillier, André Félibien, S.18).

149 Cosnac, Richesses, S.254. Zu den englischen Nachlassverkäufen der Zeit siehe: Michel, Mazarin, S.241. 
feld Nicolas Fouquets eingeordnet werden muss, ohne dass ersichtlich wäre, wer es in Auftrag gegeben hat. Die um 1651 angefertigte »Heilige Familie« Poussins wurde wahrscheinlich für Marie-Madeleine de Castille noch vor ihrer Hochzeit mit Fouquet erschaffen.

Die Anweisungen Nicolas', die aus den Briefen seines Bruders herausgelesen werden können, lassen vermuten, dass Fouquet keine spezifischen Neigungen auf dem Gebiet der Kunst verfolgte. Es ging ihm vielmehr darum, große und dekorative Gemälde für die Einrichtung seines neuen Schlosses zu erhalten ${ }^{150}$. Obgleich diese Indifferenz gegenüber Themen und Künstlern und das Insistieren auf dem dekorativen Wert der Gemälde den vorzugsweisen Kauf von günstigen Kopien (in den Sammlungen des 17. Jahrhunderts weit verbreitet) zur Folge hätte haben können, finden sich in der Sammlung Fouquets überwiegend Originale. Fouquets Gemäldesammlung war zwar klein, aber fein. Im Hinblick auf den Aspekt der sozialen Distinktion ist ihre Zusammensetzung daher umso auffälliger. Seine Sammlung umfasste allein neun Bilder der Familie Bassano, die auch von Mazarin heiß begehrt wurden, dessen Sammlung elf Bassanos einschloss. Bedenkt man die geringe Größe seiner Sammlung (im Gegensatz zu Mazarins Kollektion, die rund 858 Gemälde umfasste), ist es bemerkenswert, dass Fouquet eine so große Anzahl an Bildern der von Mazarin hochgeschätzten Künstlerfamilie in seine Sammlung aufnahm. Belegt werden kann auch der Besitz von drei Gemälden aus der Hand Paolo Veroneses - ein von den französischen Sammlern (besonders von Mazarin) ebenfalls hochbegehrter Maler. Obwohl 1655, als Louis in Rom Gemälde für seinen Bruder erwarb, der Marktwert der Bilder aus der Hand von Poussin extrem hoch war, finden sich in der Sammlung des Finanzministers vier Gemälde des in Rom lebenden Franzosen, darunter zwei Kopien, die aber von den Zeitgenossen wie Originale behandelt wurden.

\subsubsection{Die Tapisserien}

Bis zum Bau von Vaux-le-Vicomte ist wenig über die Tapisseriesammlung des Finanzministers bekannt. Nur vereinzelte Käufe von Teppichen scheinen von Louis Fouquet in Rom getätigt worden zu sein, darunter ein Perserteppich, den der Abbé in seiner Beschreibung der Sendung vom Januar 1656 ohne nähere Erläuterung aufführt ${ }^{151}$. Ein Inventar des Mobiliars von Saint-Mandé fehlt. Man muss jedoch davon ausgehen, dass auch hier - besonders anlässlich der herrschaftlichen Empfänge - Tapisserien die Wände schmückten. Das Inventar seines Appartements im Louvre führt eine »vieille verdure« aus der Auvergne (15. oder 16. Jahrhundert?) und einen ebenfalls alten Wandteppich

150 »Comme j'ai prévu que ce seroit un de vos souhaits, je ne cherche que de grands tableaux, propres à grands appartements « (Louis Fouquet in einem Brief an Nicolas Fouquet vom 23. August 1655, in: FouQuet, Lettres, S. 295).

151 Louis Fouquet in einem Brief an Nicolas Fouquet vom 11. Januar 1656, in: ibid., S. 298 . 
aus Flandern auf ${ }^{152}$. Erst in Vaux-le-Vicomte wird der Finanzminister den Erwerb der kostbaren Tapisserien an königliche Maßstäbe heranführen und den Bestrebungen Heinrichs IV. nacheifern, der durch eine gesetzliche Verfügung versucht hatte, das Bildwirkerhandwerk in Frankreich zu etablieren.

In den Sammlungen von Saint-Mandé begegnen sich Herkunft und Zukunft des aufstrebenden Finanzministers. Die Bibliothek und die Kuriositätensammlung blieben dem geistigen Erbe seines Elternhauses und damit zugleich dem Habitus der bourgeoisie de robe verpflichtet. Die Vielzahl geographischer und historischer Werke, über die das Inventar der Bibliothek Auskunft gibt, folgte ganz den Vorlieben François Fouquets, der die Geschäftsinteressen der Familie verstärkt auf den Überseehandel gelenkt hatte. Die Interessen von Nicolas' Mutter fanden sich sowohl in den zahlreichen Werken zu Religion und Religionsgeschichte in der Bibliothek als auch in der durch Pecquet vertretenen medizinischen Literatur wieder.

Das Sammeln von Skulpturen, Gemälden und Tapisserien in größerem Stil hingegen hatte der Finanzminister nicht in seinem Elternhaus kennengelernt. In seinem Verhalten als Sammler richtete sich Nicolas Fouquet nach den Konventionen der anderen Minister. Besonders an der Gemäldesammlung ist eine Orientierung an der Kollektion Mazarins selbst nicht zu übersehen.

\section{Literaturpatronage und Preziosität Fouquet in Dichterkreisen}

Im 17. Jahrhundert war es in der haute bourgeoisie und der noblesse de robe eine weit verbreitete Sitte, sich mit der Förderung der Literatur mäzenatisch zu betätigen. Diese Art von Patronage beruhte auf einem gegenseitigen Interesse. Dem Gönner brachte sie gesellschaftliches Ansehen, den Literaten die Aussicht auf eine auskömmliche finanzielle Unterstützung. Durch Buchwidmungen versuchten die Dichter, die Aufmerksamkeit potenzieller Patrone zu gewinnen. Dabei waren »der Rang eines Großen, sein Einfluss in der Gesellschaft, seine finanzielle Lage [...] für die meisten Autoren die entscheidenden Gesichtspunkte bei der Wahl ihrer Gönner «153. Fand ihr Ansinnen Gehör, so wurden sie durch eine finanzielle Unterstützung in Form von Pensionszahlungen, Gratifikationen oder kostenloser Logis belohnt, auf die wiederum eine literarische Lobpreisung an den Gönner folgen konnte, so dass Patron und

152 »Procès verbal du scellé apposé par Monsieur le Lieutenant Civil, dans la Maison, où demeuroit Monsieur Foucquet à Paris, et à son appartement du Louvre, avec l'Inventaire de ce qui s'y est treuvé« (BN ms. fr. 7620, fol.155-190). Das Pariser Hôtel war nahezu unmöbliert, das Inventar verzeichnet keine Tapisserien. Diese waren - zusammen mit den anderen Ausstattungsstücken - anlässlich des Festes vom 17. August 1661 nach Vaux gebracht worden (FouQueT, Euvres, Bd. VI, S.137).

153 LeINER, Widmungsbrief, S. 205. 
Künstler sich in dem Bedürfnis nach gesellschaftlicher Anerkennung und sozialer Absicherung aneinander banden und in einem wechselseitigen Verhältnis standen.

Die beständigste Förderung hatten die Dichter seit den frühen 1630er Jahren durch den Premierminister Richelieu erfahren. Die auf sein Geheiß an einen großen Kreis von Literaten gezahlten königlichen Pensionen brachten den Poeten finanzielle Sicherheit, und die Gründung der Académie française schuf eine Institution, die einem Teil von ihnen einen festen Lebensunterhalt garantierte. Der Tod Richelieus im Dezember 1642 löste eine Krise des literarischen Patronagesystems aus ${ }^{154}$. Die königlichen Pensionen wurden unter dem neuen Premierminister ausgesetzt; bis in die späten fünfziger Jahre beschränkte sich Mazarins Literaturpatronage auf die Unterstützung einiger weniger Lieblingsdichter ${ }^{155}$. Die gesellschaftliche und politische Krise, die in der Fronde zu einem offenen Ausbruch kam, verschärfte die Not der Literaten, indem sie eine weitere Gruppe von Mäzenen, den Schwertadel, als Förderer der Dichter und Denker in den Hintergrund treten ließ. Es waren nun die nach gesellschaftlicher Etablierung strebenden Parlamentsräte und Financiers, von denen sich die Dichter finanzielle Unterstützung erhofften.

Der in sein neues Amt erhobene Nicolas Fouquet stellte in den Augen der Literaten einen optimalen Patron dar. Als langjähriger Salonbesucher war Fouquet mit weiten Kreisen der literarischen Welt persönlich bekannt. Als Finanzminister verstand er es, Geldmittel zu organisieren und zu verteilen. So kann es nicht verwundern, dass mit der Übernahme der surintendance die Zahl der dem Finanzminister und seiner Frau gewidmeten Bücher schlagartig anstieg. Schnell sprach sich zweifellos herum, dass die Pensionszahlungen Fouquets relativ hoch ausfielen. Für die Dichter war er ein idealer und attraktiver Patron, der ihre Dedikationen erhörte und ihre finanziellen Wünsche über ihre Erwartungen hinaus erfüllte ${ }^{156}$. Die den Poeten bewilligten Zahlungen waren von unterschiedlicher Höhe: Sie lagen zwischen 400 und 2000 Livres und entsprachen damit den königlichen Pensionen der Zeit vor Mazarin $^{157}$. Doch nicht allein die Dichter profitierten von einer Bindung an den neuen Finanzminister. Für Fouquet hatte das Ende der Fronde ein hohes Amt gebracht, das er sichern und ausbauen musste. Die Verbreitung seines Namens durch die Literaten war für ihn ein willkommenes Mittel, seine neue

\footnotetext{
154 Ranum, Artisans of Glory, S.157f.; Bannister, Crisis, S.18-21.

155 Ibid., S.18. Die Enttäuschung der Dichter über das geringe Engagement Mazarins machte sich während der Fronde in der Vielzahl der gegen den exilierten Premierminister gerichteten »mazarinades « Luft.

156 Ibid., S. 19.

157 Laut Bannister (ibid.) soll Scarron von Fouquet im Jahr 1653 eine Pensionszahlung in Höhe von 1600 Livres erhalten haben; Collinet, La Fontaine, S. 274 zufolge erhielt La Fontaine jährliche Zahlungen in Höhe von 1000 Livres. Vergleicht man diese Summen mit den von Mazarin gezahlten Pensionen von 50-60 Livres, so versteht man die finanzielle Not und den Unmut der Dichter unter dem neuen Premierminister.
} 
Stellung publik zu machen und sich als surintendant des Finances zu etablieren. Zu Beginn seiner Amtszeit war es ein noch kleiner Kreis von Literaten, den der Finanzminister an sich band. Mit der Zeit aber wurde dieser zu einem Hof von literarischen Gefolgsleuten und Fouquet zu dem Mann, der die Krise der Literaturpatronage im vorludovizianischen Frankreich zu überwinden vermochte ${ }^{158}$.

Paul Scarron, der in den 1620er Jahren in den literarischen Zirkeln von Paris verkehrte, wo er Colletet sowie die Geschwister Scudéry kennenlernte und mit Françoise d'Aubigné (der späteren Mme de Maintenon), die er 1652 ehelichte, in seinem Haus in der Rue Neuve-Saint-Louis im Marais einen Salon unterhielt, war zeitlebens in Geldnot. Er ist der erste Dichter, von dem Buchwidmungen an die Familie Fouquet bekannt sind.

Die dem zweiten Teil seines »Roman comique « vorangestellte Dedikation aus dem Jahre 1654 ist an Marie-Madeleine de Castille adressiert, die seiner Gattin freundschaftlich verbunden war.

Vous êtes belle sans être coquette;

Vous êtes jeune sans être imprudente;

Et vous avez beaucoup d'esprit sans ambition de le faire paraître,

Vous êtes vertueuse sans rudesse,

pieuse sans ostentation,

riche sans orgueil,

et de bonne maison sans mauvaise gloire ${ }^{159}$.

Mit dieser Widmung wurde Scarron zum ersten Poeten, der von Fouquet eine Gratifikation erhielt. In seinen vor $1656 \mathrm{zu}$ datierenden Briefen an Fouquet und dessen Bruder ${ }^{160}$ spricht er die Unterstützung durch den surintendant sowie seine Freigebigkeit direkt an:

Il n'appartient qu'à ceux de votre Maison de porter la générosité, \& la bonté aussi loin qu'elle peut aller. Monsieur le Procureur General votre frere, m'a donné une pension sans que je lui aye demandée, \& vous m'estes venu voir sans que j'aye brigué l'honneur de votre visite: une telle bonté me donne à vous terriblement, pour parler à la mode ${ }^{161}$.

Die Pensionszahlungen durch Fouquet müssen Scarrons Erwartungen weit übertroffen haben, denn an den surintendant schreibt er: »Je voudrois donc bien que quelqu'un vous dit pour moi; que vous estes le plus genereux homme

158 Fouquet ist in den Augen Bannisters der Überwinder der Krise der Literaturpatronage, die nach dem Tod Richelieus eingesetzt hatte. Durch die breite Förderung von Literaten brachte der Finanzminister zum ersten Mal seit 1642 einem Großteil der Dichter finanzielle Sicherheit, so dass Bannister zu dem Schluss kommt: »He was the reincarnation of Richelieu « (BANNISTER, Crisis, S.19).

159 LeINER, Widmungsbrief, S. 108.

160 Die Briefe Scarrons finden sich in den »Dernières Euvres de M. Scarron« (La Haye, 1730). Die chronologische Datierung der Briefe beginnt auf der Seite 124 mit dem 17. März 1656.

161 SCARron, Dernières CEuvres, S. 81 (Datum und Adressat werden nicht genannt). 
du monde, \& que toutes les graces que vous me faites, vont toujours au delà de toutes les prieres que je vous faits $« 162$.

Die zweite Widmung Scarrons, die zugleich die einzige Dedikation an den Finanzminister selbst ist, ging der Komödie »Le Gardien de soi-même« voraus, die am 14. Juli 1655 erschien. In einem Brief dankt Scarron dem Finanzminister für das Wohlwollen gegenüber seinem Werk: »La grace que vous m'avez faite de ne mépriser pas la Comédie que je vous ai dédiée, m'obligeois assez à me donner à vous, sans que vous eussiez à m'y engager davantage par une nouvelle obligation ${ }{ }^{163}$. Auch im folgenden Jahr gedachte der Dichter seines Förderers, indem er ihm die burleske Ode »Léandre et Héros « widmete.

Madeleine de Scudéry gehörte ebenfalls zu den Literaten, die bereits Mitte der fünfziger Jahre durch Fouquet finanzielle Unterstützung fanden. 1655 dankte Paul Pellisson ihm als Freund Madeleine de Scudérys im Namen Saphos ${ }^{164}$ mit dem »Remercîment du Siècle à M. Fouquet « für eine Gratifikation, die der surintendant der Autorin des Romanzyklus »Artamène ou le Grand Cyrus « hatte zukommen lassen ${ }^{165}$. Darin beschreibt Pellisson das finanzielle Schicksal seiner Freundin und rühmt die Einzigartigkeit der Unterstützung durch den Finanzminister:

Sapho n'avoit point de Fortune.

Le Sort qui n'a nulle raison

Ayant renversé sa maison,

La laissoit sans autre avantage

Que son Esprit et son courage.

Dans la France de bout en bout,

On l'estimoit, mais c'estoit tout.

Le seul Foucquet ce Magnanime

Joignit les Effects à l'Estime ${ }^{166}$.

Die Bildung eines wenngleich noch kleinen Kreises von Literaten um Fouquet in den ersten Jahren nach seiner Amtsübernahme wäre undenkbar gewesen ohne die selbst vom König ${ }^{167}$ für ihre Schönheit und ihren Esprit gerühmte

162 Scarron in einem undatierten Brief an Nicolas Fouquet, in: ibid., S. 89.

${ }^{163}$ Scarron in einem undatierten Brief an Nicolas Fouquet, in: ibid., S. 94. Da Scarron in diesem Brief seine Komödienwidmung an Fouquet anspricht, die am 14. Juli 1655 erschien, kann er auf die Zeit nach dem Sommer 1655 datiert werden.

164 Madeleine de Scudéry, die in ihren Werken die Zeitgenossen unter einem jeweiligen Pseudonym nachzuzeichnen suchte, hatte sich selbst in dem Romanzyklus "Artamène ou le Grand Cyrus« den Namen Sapho gegeben, den sie fortan als literarisches Pseudonym verwendete.

165 NIDERST, Scudéry, S. 303: »Le surintendant ayant fait payer cinq cents écus à Madeleine de Scudéry «.

166 Bibliothèque de La Rochelle Ms. 672, fol. 85r.-v.

167 In einem Brief an Falconet vom 12. November 1660 schreibt Guy Patin: »Le roi aime assez jouer, mais il ne joue pas trop bien, et perd beaucoup: passe pour lui, il est le maistre, il a de quoi perdre et de quoi enrichir; il y a deux femmes à la cour avec lesquelles le roi n'a pas regret de s'entretenir et de jouer: ce sont la comtesse de Soissons, nièce de Son Eminence et $\mathrm{M}^{\text {me }}$ Foucquet, femme de $\mathrm{M}$. le procureur général et surintendant des finances« (Patin, Correspondance, S.342). 
zweite Frau Nicolas': Marie-Madeleine de Castille. Diese begann kurz nach der Hochzeit im Jahre 1651, den Zirkel um ihren Gatten durch die Aufnahme von femmes de lettres wie Marie de Béthune Charost oder Mme de Sévigné zu erweitern; die Freundschaft mit Françoise d'Aubigné ${ }^{168}$ brachte einen sehr engen Kontakt mit den Dichterkreisen und mit Paul Scarron selbst ${ }^{169}$. Die rege Verbindung Mme Fouquets mit den Poeten nährte in ihnen die Hoffnung, durch ihre Vermittlung eine Förderung des Finanzministers zu erhalten. Ihr wurden aus diesem Grund nach 1653 zahlreiche Bücher gewidmet (siehe Tabelle 2, S.161).

Mit der Förderung der Dichter folgte der bibliophile Minister nicht zuletzt einer lang gehegten Leidenschaft, denn als eifriger Salonbesucher in Paris hatte er bereits Bekanntschaft mit zahlreichen Poeten gemacht.

Die Salonkultur der ersten Hälfte des 17. Jahrhunderts war ein wichtiger Bestandteil des gesellschaftlichen Lebens von Paris. Sie wurde geprägt von dem Ideal der »grâce et de la joie ${ }^{170}$. Dichtung, Theater, Musik und selbst die Architektur der Zeit ließen sich von ihm beeinflussen und begaben sich auf die Suche nach dem einfachen Glück, nach Anmut, Reinheit und Sanftmut. Sie zeichneten sich damit klar ab gegen die Gruppe der den alten Idealen verhafteten Dichter - unerschütterliche Verteidiger der belles-lettres, der Ordnung und Harmonie, der Repräsentanten der Ästhetik der raison et de la grandeur wie Malherbe, Guez de Balzac und Chapelain ${ }^{171}$. Bereits unter Ludwig XIII. begann eine neue Art der Dichtung sich in den femininen Zirkeln und Salons ${ }^{172} \mathrm{zu}$ entfalten, unter denen derjenige der Mme de Rambouillet der berühmteste war. Als dichterischer Mittelpunkt der ruelle galt Vincent Voiture, dessen Werk der poésie galante verpflichtet war und der die Troubadour-Dichtung wieder aufleben ließ. Daneben gab es andere Salons, wie jener der Madeleine de Souvré, Marquise de Sablé, oder der Salon der Mme de Brégy.

Die Fronde hatte den Siegeszug der Salonkultur nicht aufgehalten: Mme de La Fayette und Mme de La Suze mit ihren Salons, aber auch der Poet Gilles Ménage mit seinen in der Mitte der Woche stattfindenden Mercuriales und nicht zuletzt Madeleine de Scudéry, bei der sich die salonniers in den »samedis « zusammenfanden, ließen die neue Form des literarischen Forums weiterleben. An den Zusammenkünften nahmen auch Dichter und Gelehrte teil,

\footnotetext{
168 Seit 1652 Mme Scarron.

169 Die Popularität Mme Fouquets vor allem in Künstlerkreisen zeigte sich nicht zuletzt darin, dass sie ab 1654 selbst Adressatin von Widmungsbriefen wurde.

170 AdAm, Autour de Fouquet, S. 284.

171 Vgl. Petitfils, Fouquet, S. 260.

172 Ein Salon ist ein Ort, an dem sich der französische Adel fern des Königshofes trifft, um Konversation zu betreiben und zu spielen. Im Gegensatz zu den Salons des 16. Jahrhunderts, die in relativer Nähe zum Hof existierten, definiert sich die Salonkultur des 17. Jahrhunderts in Abgrenzung zum Königshaus. Der Begriff »Salon«, erst seit 1793 gebräuchlich, ist nicht klar abgegrenzt.
} 
lasen aus ihren Werken vor und präsentierten sie hier nicht selten erstmalig der Öffentlichkeit. Bei den Treffen handelte es sich keineswegs um geschlossene Gesellschaften. Die Durchlässigkeit der einzelnen Kreise inspirierte Maucroix zu einer unvollendet gebliebenen Komödie, in der es heisst: »C'est une bagatelle, en moins d'un mois entier, Vous ferez connoissance avec tout le quartier: Connoissance à Paris est chose bien facile, Et l'on connoît bientôt et la cour et la ville ${ }^{173}$.

Bereits seit Beginn seiner politischen Laufbahn verkehrte Fouquet in den Pariser Salons, wo er auf Dichter und Denker traf und selbst Reime und Rätsel vortrug. Häufiger Gast war er in dem Salon von Suzanne de Bruc, Marquise du Plessis-Bellière, die, umgeben von ihren Brüdern und ihrer Nichte, in ihrem Haus in Charenton, unweit von Saint-Mandé, viele Gäste empfing. Im Salon der Marquise sollten auch Fouquets dichterische Versuche Gehör finden.

Ende 1653 ereigneten sich zwei Todesfälle, von denen der erste die Salongesellschaft zutiefst aufwühlte: der Papagei der Salondame starb ${ }^{174}$, und die Trauer um ihn nahm solche Ausmaße an, dass der Tod des Ehemannes ein paar Monate später fast unbemerkt blieb. Das Ableben des gefiederten Haustieres Mélinthe löste eine Sturzflut von Sonetten und bouts-rimés aus, allen voran ein Gedicht aus der Feder Nicolas Fouquets:

Plutôt le procureur maudira la chicane,

Le joueur de piquet voudra se voir capot,

Le buveur altéré s'éloignera du pot

Et tout le Parlement jugera sans soutane;

On verra Saint-Amant devenir diaphane

Le goutteux tout perclus hantera le tripot,

Mme de Rohan quittera son Chabot

Et d'ouïr le sermon sera chose profane.

Un barbier pour raser ira sans coquemar,

Le clocher de Saint-Paul sera sans jacquemar,

L'évêque grenoblois fera couper sa barbe,

Que d'oublier jamais ton funeste débris

Aimable perroquet: j'en jure Sainte-Barbe,

Ton portrait à jamais ornera mon lambris ${ }^{175}$.

Ironische Sonette dieser Art hatten in den Salons einen ungeheuren Erfolg; Loret, Benserade, Boisrobert - alle versuchten den Tod des Papageien mit kecken Reimen zu kommentieren: »[Le sonnet de Fouquet réveilla] tout ce

173 Zitiert nach: Chatelain, Foucquet, S. 87.

174 Der Bericht vom Tod des Papageien findet sich in der "Muze historique« vom 29. November 1653 (Bd. I, Lettre XLV, S. 434f.).

175 Chatelain, Foucquet, S.73f. weist darauf hin, dass lange daran gezweifelt wurde, ob das Sonett tatsächlich aus der Feder Fouquets stammt; Details, wie der Bezug zu der Fouquet bekannten Familie Rohan oder Pierre Scarron, dem Bischof von Grenoble, sowie eine Notiz Pellissons über dem Sonett, schienen jedoch Fouquet als Autor zu bestätigen. 
qu'il y avait de gens en France qui savaient rimer et l'on ne vit jamais durant quelques mois que des sonnets sur les mêmes bouts-rimés «176.

In der älteren Literatur hat man gelegentlich angenommen, dass der Finanzminister und seine Frau in ihren Häusern selbst einen Salon unterhalten hätten. Es gibt jedoch keine Memoiren oder Briefe, die von regelmäßigen Zusammenkünften in den Pariser hôtels der Fouquets, in Saint-Mandé oder gar in Vaux-le-Vicomte berichten ${ }^{177}$. Bei den Literaten finden wir jedoch Andeutungen, dass die Häuser Fouquets den Dichtern offenstanden:

Chacun sait que ce grand ministre n'est pas moins le surintendant des belles-lettres que des finances; que sa maison est aussi ouverte aux gens d'esprit qu'aux gens d'affaires; et que soit à Paris, soit à la campagne, c'est dans les bibliothèques qu'on attend ces précieux moments qu'il dérobe aux occupations qui l'accablent, pour en gratifier ceux qui ont quelque talent d'écrire avec succès ${ }^{178}$.

Und in dem Romanzyklus »Clélie« von Madeleine de Scudéry finden wir den Hinweis darauf, dass man bei den Fouquets zusammenkam, um Konversation zu betreiben: »[Scarus (=Scarron) écrit] mille choses spirituelles \& divertissantes, qui servent au plaisir de l'illustre Cléonime [Fouquet] \& de qui enfin la conversation est recherchée d'un fort grand nombre de personnes de qualité qui sont tres-souvent chez luy « ${ }^{179}$. Belegt sind darüber hinaus unzählige Feste, die in den Häusern der Fouquets veranstaltet wurden. Hiervon berichten die Journale der Zeit, allen voran die $»$ Muze historique ${ }^{180}$. Von nahezu allabendlichen Spielen im Kreise um Mme Fouquet erzählt Gourville in seinen »Mémoires«:

On jouait presque tous les jours chez Mme Foucquet assez gros jeu; Mme de LaunayGravé, depuis marquise de Piennes, y jouait ordinairement avec un nombre d'autres dames, et quelquefois aussi des messieurs. [...] Ces jeux-là se jouaient sans avoir l'argent sur table; mais, à la fin du jeu, on apportait une écritoire: chacun écrivait sur une carte ce qu'il devait à l'autre, et, en envoyant la carte, on apportait l'argent ${ }^{181}$.

176 Pellisson, zitiert nach: PetrTfiLs, Fouquet, S. 263. Insgesamt entstanden circa 28 Sonette, die den Tod des Papageien zum Thema hatten - der Tod des Ehemannes der Marquise hingegen inspirierte nur einen einzigen Dichter zu einem Sonett (abgedruckt in: CHATELAIN, Foucquet, S.71, Anm.3). Für Fouquet gehörte das Verfassen von kleinen Versen seit der Schulzeit zu einem Zeitvertreib, dem er sich in ruhigen Stunden hingab. Er schrieb Änigmen, galante Sonette, Madrigale in lateinischer oder französischer Sprache, die vom Jesuitenpater Deschampsneufs gesammelt wurden (CHATELAIN, Foucquet, S. 77).

177 Chatelain (ibid., S. 270) behauptet jedoch, dass sich regelmäßig eine Gruppe um Mme Fouquet zusammengefunden habe: »Mazarin ayant exigé [...] que le surintendant vînt demeurer plus près du Louvre. [...] Mme Fouquet prit possession de l'hôtel de son beaufrère M. de Narbonne. [...] C'est là que la cour des poètes vint la trouver plus nombreuse et plus empressée que jamais«.

178 Pierre Corneille, CEdipe, Avis au lecteur, in: Corneille, Euvres, S.124f.

179 SCUDÉrY, Clélie, Bd. X, S. 1226-1228.

180 Siehe unter anderem Kapitel 4.

181 Gourville, Mémoires, S. 123. 
Definiert man einen Salon nicht als einen Ort regelmäßiger Zusammenkünfte, bei denen literarische Gespräche geführt wurden ${ }^{182}$, so kann man auch die Gesellschaften, die sich um Mme Fouquet zusammenfanden, als einen Salon bezeichnen. In jedem Fall wurde im Hause Fouquet oft gespielt und Konversation getrieben. Bedenkt man Fouquets Freude am Reimen und Rätseln, dann ist nicht auszuschließen, dass in seinem Kreis auch Literatur vorgetragen und diskutiert wurde. Über die Regelmäßigkeit dieser Zusammenkünfte sowie die anwesenden Gäste gibt es jedoch nur sehr spärliche Informationen ${ }^{183}$. Um einen preziösen Salon im Sinne der Marquise de Rambouillet oder Madeleine de Scudéry, um einen Salon als literarischen Ort also, scheint es sich allerdings nicht gehandelt zu haben, denn es sind weder literarische Erzeugnisse aus ihm hervorgegangen (wie zum Beispiel im Falle der Chambre bleue die »Guirlande de Julie« oder die Romanzyklen der Madeleine de Scudéry, die als verschlüsselte Salonchroniken und Konversationsmemoiren ihres Salons angesehen werden ${ }^{184}$ ), noch rekurrieren die Dichter in ihren Widmungen und Lobgedichten an Fouquet und seine Frau auf die Zusammentreffen in den Salons.

Die Kulturpatronage der ersten Phase durch Nicolas Fouquet ist der Erfüllung einer gesellschaftlichen Norm verpflichtet. $\mathrm{Zu}$ Beginn seiner Karriere musste sich der noch junge Aufsteiger in seiner neuen Position etablieren und behaupten. Seine Schlossanlage in Saint-Mandé bot ihm den seiner Stellung entsprechenden repräsentativen Rahmen, der durch die Sammlungen vervollständigt wurde. Schon bald nach der Übernahme des neuen Amtes änderten sich jedoch mit der politischen Lage auch die kulturpolitischen Absichten Nicolas Fouquets.

182 Bei den bekannten Salons, wie etwa der Chambre bleue der Mme de Rambouillet oder, nach der Fronde, den »samedis« der Madeleine de Scudéry wissen wir von einer Regelmäßigkeit der Zusammenkünfte, von denen im Falle Saphos bereits die Bezeichnung für ihren Zirkel spricht.

183 Vor allem in den Memoiren von Gourville finden sich Angaben zu Treffen bei den Fouquets; zum Beispiel: Gourville, Mémoires, S.123f. Hier werden auch die Namen einzelner Gäste genannt.

184 In den Romanen der Scudéry finden sich neben Binnennovellen, die die in den Salons erlebten Ereignisse aufgreifen, chiffrierte Porträts der Salonbesucher. 